J. DIFFERENTIAL GEOMETRY

54 (2000) $177-225$

\title{
A HODGE THEORY FOR SOME NON-COMPACT MANIFOLDS
}

\author{
ZULFIKAR M. AHMED \& DANIEL W. STROOCK
}

\section{Introduction}

Suppose that $M$ is a differentiable manifold, let

$$
\Omega^{*}(M)=\bigoplus_{q=0}^{\operatorname{dim}(M)} \Omega^{q}(M)
$$

be the smooth diffential forms on $M$, and use $d: \Omega^{*}(M) \longrightarrow \Omega^{*}(M)$ to denote the exterior derivative operator. Then the renowned theorem of De Rham says that, if

$$
H^{0}(M) \equiv\left\{\omega \in \Omega^{0}(M): d \omega=0\right\}
$$

and, for $1 \leq q \leq \operatorname{dim}(M)$,

$$
H^{q}(M) \equiv\left\{\omega \in \Omega^{q}(M): d \omega=0\right\} /\left\{d \omega: \omega \in \Omega^{q-1}(M)\right\}
$$

then $H^{q}(M)$ can be identified as the $q$ th $\check{C}$ ech cohomology space of $M$ over $\mathbb{R}$. Given a closed form $\omega \in \Omega^{q}(M)$, we will use $[\omega]$ to denote its De Rham equivalence class. That is, $[\omega]=\{\omega\}$ if $q=0$ and $[\omega]=$ $\omega+d \Omega^{q-1}$ if $q>1$.

Next, assume that $M$ is compact, endow $M$ with a Riemannian structure, and use $\lambda_{M}$ to denote the associated Riemannian measure. One interpretation of Hodge's refinement to De Rham's theorem is that

Received February 10, 2000, and, in revised form, June 12, 2000. Both authors were partially funded by support from NSF grant DMS9625782. 
it asserts the existence, for each closed $\omega \in \Omega^{q}(M)$, of a unique $\eta \in[\omega]$ with minimal $L^{2}\left(\lambda_{M} ; \Lambda^{q}\left(T M^{*}\right)\right)$-norm. In particular, Hodge's result provides a canonical (modulo the Riemannian structure) procedure for selecting De Rham representatives.

Of course, the structure of Hodge's theory is much richer than the preceding indicates. Indeed, the proof of Hodge's theory leads, via elementary variational calculus, first to the introduction of the formal adjoint $\delta=d^{* \lambda_{M}}$ of $d$ in $L^{2}\left(\lambda_{M} ; \Lambda^{*}\left(T M^{*}\right)\right)$, then to the Hodge operator

$$
H=(d+\delta)^{2}=d \delta+\delta d
$$

and finally to the realization that everything comes down to showing that each $\omega \in \Omega^{*}(M)$ admits a Hodge decompostion:

$$
\omega=\eta+d \xi_{1}+\delta \xi_{2}
$$

for some $\xi_{1}, \xi_{2} \in \Omega^{*}(M)$ and a unique $H$-harmonic $\eta \in \Omega^{*}(M)$ (i.e., $H \eta=0)$.

One way to prove Hodge's decomposition theorem is to study the Cauchy initial value problem for the parabolic equation

$$
\partial_{t} \omega_{t}+H \omega_{t}=0 \quad \text { on }(0, \infty) \times M \quad \text { with } \quad \omega_{0}=\omega \in \Omega^{*}(M) .
$$

Indeed, because the action of $H$ is seen in local coordinates to be a system in which the row corresponding to $I=\left(i_{1}, \ldots, i_{q}\right)$ looks like

$$
-\Delta \omega_{I}+\sum_{J} R_{I, J}^{0} \omega_{J}
$$

where the $R_{I, J}^{0}$ 's are various components of the Riemann curvature tensor $R$, one can easily check, via the matrix-valued version of the Feynman-Kac formula (cf. [11]), that the semigroup of operators $\mathbf{Q}_{t}^{0}=$ $e^{-t H}$ which solve (0.1) (in the sense that $\omega_{t}=\mathbf{Q}_{t}^{0} \omega$ ) is just as bounded as the scalar heat flow semigroup of operators $\mathbf{P}_{t}^{0}=e^{t \Delta}$. In particular, each $\mathbf{Q}_{t}^{0}$ determines a unique extension as a self-adjoint contraction $\overline{\mathbf{Q}_{t}^{0}}$ on $L^{2}\left(\lambda_{M} ; \Lambda^{*}\left(T M^{*}\right)\right)$. Moreover, because $\lambda_{M}$ is finite and the heat kernel is bounded, it is obvious that each $\overline{\mathbf{Q}_{t}^{0}}$ is Hilbert-Schmidt, and therefore compact.

Now let $-\bar{H}$ denote the generator of the semigroup $\left\{\overline{\mathbf{Q}_{t}^{0}}: t>0\right\}$, and use elementary spectral theory to see that

$$
\operatorname{ker}(\bar{H})=\operatorname{ker}\left(I-\overline{\mathbf{Q}_{1}^{0}}\right) .
$$


Because $\overline{\mathbf{Q}_{1}^{0}}$ is compact, and therefore $I-\overline{\mathbf{Q}_{1}^{0}}$ is Fredholm, (0.2) already shows that $\operatorname{ker}(\bar{H})$ is finite dimensional. In addition, it (together with a little elliptic regularity theory) shows that if $\eta$ is the orthogonal projection of $\omega \in \Omega^{*}(M)$ onto $\operatorname{ker}(\bar{H})$, then $\eta \in \Omega^{*}(M)$ and $\omega-\eta=\zeta-\mathbf{Q}_{1}^{0} \zeta$ for some $\zeta \in \Omega^{*}(M)$. But

$$
\begin{aligned}
\zeta-\mathbf{Q}_{1}^{0} \zeta & =-\int_{0}^{1} \partial_{\tau} \mathbf{Q}_{\tau} \zeta d \tau=H \int_{0}^{1} \mathbf{Q}_{\tau} \zeta d \tau=d \xi_{1}+\delta \xi_{2}, \quad \text { where } \\
\xi_{1} & =\delta \int_{0}^{1} \mathbf{Q}_{\tau} \zeta d \tau \text { and } \xi_{2}=d \int_{0}^{1} \mathbf{Q}_{\tau} \zeta d \tau
\end{aligned}
$$

Equivalently, $\omega=\eta+d \xi_{1}+\delta \xi_{2}$ is the Hodge decompositon of $\omega$.

We now want to see how much of the preceding survives when we drop the assumption that $M$ is compact. Obviously, not much! Indeed, when $M$ is not compact, it is rare for the operators $\mathbf{P}_{t}^{0}$ to be compact, and therefore even less likely that the operators $\mathbf{Q}_{t}^{0}$ will be. ${ }^{1}$ On the other hand, things start looking less bleak when we replace the Riemannian measure $\lambda_{M}$ by the measure $m^{U}(d x)=e^{-U(x)} \lambda_{M}(d x)$, where we choose $U: M \longrightarrow[0, \infty)$ to grow so fast at infinity that $m^{U}$ is finite. At the same time, we replace the original Hodge operator $H=\delta d+d \delta$ by the weighted Hodge operator $H^{U} \equiv \delta^{U} d+d \delta^{U}$, where $\delta^{U}$ is the formal adjoint of $d$ computed this time relative to $m^{U}$. That is, $\delta^{U} \omega=e^{U} \delta\left(e^{-U} \omega\right)$. For one thing, at least the reference measure is now finite. Secondly, but less immediately apparent, is the fact that, by making $U$ grow fast enough, one can force the operators in the semigroup $\left\{\mathbf{P}_{t}^{U}: t>0\right\}$, which now plays the role that $\left\{\mathbf{P}_{t}^{0}: t>0\right\}$ did before, to be compact.

More precisely, in local coordinates, $H^{U} \omega$ takes the form

$$
\left(H^{U} \omega\right)_{I}=-L^{U} \omega_{I}+\sum_{J} R_{I, J}^{U} \omega_{J}
$$

where

$$
L^{U} \varphi \equiv e^{U} \operatorname{div}\left(e^{-U} \operatorname{grad} \varphi\right)=\Delta \varphi-\langle\operatorname{grad} U, \operatorname{grad} \varphi\rangle
$$

and the $R_{I, J}^{U}$ 's are built out of the $R_{I, J}^{0}$ 's and the Hessian of $U$. Ignoring, for the moment, the problems caused by the $R_{I, J}^{U}$ 's, let $\left\{\mathbf{P}_{t}^{U}: t>0\right\}$ be the semigroup of solution operators for the Cauchy initial value problem corresponding to the scalar heat equation $\partial_{t} u_{t}=L^{U} u_{t}$. At least when

\footnotetext{
${ }^{1}$ In fact, without further restrictions on the Riemann curvature, there is no reason to suppose that the $\mathbf{Q}_{t}^{0}$ 's will even exist in any meaningful sense.
} 
$M=\mathbb{R}^{d}$, it has been known for some time (cf. [6] and [8]) that when $U$ grows like a power strictly larger than 2 of the distance to a point, each $\mathbf{P}_{t}^{U}$ has a uniformly bounded kernel representation with respect to $m^{U}$. Following the approach in [8], as modified in [14], we show in $\S 2$ that when $U$ satisfies the condition given in Theorem 2.11, the same conclusion (cf. (2.12)) holds for any complete, connected, Riemannian manifold $M$ whose Ricci curvature of $M$ is bounded uniformly from below. Using techniques which are much more closely related to those in [6], F.-Y. Wang [17] and M. Roeckner and F.-Y. Wang [10] have also derived criteria from which it seems likely that the basic estimates in $\S 2$ follow.

In order to apply the preceding to $\mathbf{Q}_{t}^{U}$, we must impose additional conditions which guarantee that the Feynman-Kac formula does not destroy everything. For example, it is sufficient that both the Riemann curvature operator and the Hessian of $U$ (cf. (3.19), (4.4), and (4.5)) be bounded uniformly from below. That is, we can show (cf. Lemmas $5.2)$ under these conditions that $\mathbf{Q}_{t}^{U}$ extends to a self-adjoint, HilbertSchmidt contraction operator on $L^{2}\left(m^{U} ; \Lambda^{*}\left(T M^{*}\right)\right)$. In addition, we show that each $\mathbf{Q}_{t}^{U}$ maps $L^{2}\left(m^{U}\right)$ into $C_{\mathrm{b}}(M ; \mathbb{R})$, which means that $m^{U}$-square integrable $H^{U}$-harmonic forms are bounded. Knowing this, we develop in $\S 5$ (cf. Theorem 5.1) the Hodge decomposition theorem corresponding to the weighted Hodge operator $H^{U}$, and in $\S 6$ (cf. Theorem 6.4) we apply it to obtain a Hodge theory for the De Rham cohomolgy of $M$. For related results, the reader might want to consult the articles [2], [3], [7], and [17].

Throughout this article, $M$ will denote a complete, connected, finite dimensional Riemannian manifold whose Ricci curvature is bounded uniformly below by $-\kappa_{\text {Ric }}$, where $\kappa_{\text {Ric }} \geq 0$. Under these circumstances, it is known (cf. [19]) that the (unique) bounded solutions to the Cauchy initial value problem for the heat equation

$$
\partial_{t} u=\Delta u_{t} \quad \text { on }(0, \infty) \times M \quad \text { with } u_{0}=\varphi \in C_{\mathrm{b}}(M ; \mathbb{R})
$$

is given by

$$
u_{t}(x)=\left[\mathbf{P}_{t}^{0} \varphi\right](x)=\int_{M} \varphi(y) p_{t}^{0}(x, y) \lambda_{M}(d y)
$$

where the heat kernel $(t, x, y) \in(0, x, y) \times M \times M \longmapsto p_{t}^{0}(x, y) \in(0, \infty)$ is a smooth function which satisfies estimates (cf. [9]) which are strikingly like those which hold when $M=\mathbb{R}^{d}$. 
Acknowledgments. A lot of this article grew out of a casual remark made to us by Tom Mrowka ${ }^{2}$ after we told him about the sort of estimates which are given in $\S 2$. In particular, it was his remark which prompted us to think about applications to Hodge theory. The second author is also grateful to I.M. Singer, who, as always, was ready to express interest and lend support. Finally, we are grateful to Laurent Saloff-Coste who provided us with the reference to Coulhon's paper [5].

\section{A little diffusion theory}

Suppose that $U: M \longrightarrow[0, \infty)$ is a (locally) Lipschitz continuous function $^{3}$ which is continuously differentiable on an open set $D(U)$ whose complement has $\lambda_{M}$-measure 0 . Further assume that

$$
x \in D(U) \longmapsto\|\operatorname{grad} U\| \in \mathbb{R}
$$

admits a (necessarily unique) continuous extension to the whole of $M$. Finally, for some $C \in[1, \infty)$, assume that

$$
\Delta U \leq C(1+U+\|\operatorname{grad} U\|) \quad \text { in the sense of distributions. }
$$

Next, define the second order, elliptic operator $L^{U}$ on $C^{2}(M ; \mathbb{R})$ so that

$$
\begin{array}{r}
L^{U} \varphi=e^{U} \operatorname{div}\left(e^{-U} \operatorname{grad} \varphi\right)=\Delta \varphi-\langle\operatorname{grad} U, \operatorname{grad} \varphi\rangle \\
\text { where } \operatorname{grad} U \equiv \mathbf{0} \text { off of } D(U) .
\end{array}
$$

Finally, determine the measure $m^{U}$ by

$$
m^{U}(d x)=e^{-U(x)} \lambda_{M}(d x),
$$

where $\lambda_{M}$ is the Riemannian measure on $M$.

The purpose of this section is to verify the statement which follows.

Theorem 1.4. For each $x \in M$ there is a unique probability measure $\mathbb{P}_{x}^{U}$ on $\mathcal{P}(M) \equiv C([0, \infty) ; M)$ with the properties that $\mathbb{P}_{x}^{U}(p(0)=x)=1$ and, for each $\varphi \in C_{\mathrm{c}}^{\infty}(M ; \mathbb{R})$,

$$
\varphi(p(t))-\int_{0}^{t} L^{U} \varphi(p(\tau)) d \tau
$$

\footnotetext{
${ }^{2}$ In truth, Tom thought that we were dealing with the very infinite measure $e^{U} d \lambda_{M}$ instead of the very finite one $e^{-U} d \lambda_{M}$, but we have forgiven him even if he has not forgiven us.

${ }^{3}$ Although we are, in the end, most interested by $U$ 's which are smooth, our analysis forces us to consider functions which may be as badly behaved as the function which measures the distance from a fixed reference point.
} 
is a $\mathbb{P}_{x}^{U}$-martingale. ${ }^{4}$ In fact,

$$
\mathbb{P}_{x}^{U}\left(\int_{0}^{\infty} \mathbf{1}_{D(U) C}(p(t)) d t>0\right)=0,
$$

and $x \in M \longmapsto \mathbb{P}_{x}^{U} \in \mathbf{M}_{1}(\mathcal{P}(M))$ is weakly continuous. ${ }^{5}$ Moreover, if $(t, p) \in(0, \infty) \times \mathcal{P}(M) \longmapsto \bar{R}^{U}(t, p) \in(0, \infty)$ is given by (cf. (1.1))

$$
\begin{aligned}
\bar{R}^{U}(t, p) \equiv & \exp \left(\frac{1}{2} U(p(0))-\frac{1}{2} U(p(t))\right. \\
& \left.+\int_{0}^{t}\left(\frac{1}{2} C^{2}(4+U)-\frac{1}{8}\|\operatorname{grad} U\|^{2}\right)(p(\tau)) d \tau\right),
\end{aligned}
$$

then, for any bounded stopping time $e^{6} \zeta: \mathcal{P}(M) \longrightarrow[0, \infty)$,

$$
\mathbb{E}^{\mathbb{P}}{ }^{U}[F(p)] \leq \mathbb{E}^{\mathbb{P}_{x}^{0}}\left[\bar{R}^{U}(\zeta, p) F(p)\right]
$$

for all $\mathcal{B}_{\zeta}$-measurable $F: \mathcal{P}(M) \longrightarrow[0, \infty)$. Finally, if

$$
\mathbb{P}^{U}=\int_{M} \mathbb{P}_{x}^{U} m^{U}(d x),
$$

then $\mathbb{P}^{U}$ is reversible in the sense that, for each $T \in(0, \infty)$ and $\mathcal{B}_{T^{-}}$ measurable $F: \mathcal{P}(M) \longrightarrow[0, \infty)$,

$$
\mathbb{E}^{\mathbb{P}}[F(p)]=\mathbb{E}_{i}^{\mathbb{P}}\left[F\left(\check{p}^{T}\right)\right],
$$

where $\check{p}^{T}(t) \equiv p(T-t \wedge T)$.

Because (cf. $\S 6.6$ in [15]) uniqueness is a local problem and the local problem for this sort of operator is amply covered by known (cf.

\footnotetext{
${ }^{4}$ Given a probability measure $\mathbb{P}$ on $\mathcal{P}(M)$ and a measurable function $X:[0, \infty) \times$ $\mathcal{P}(M) \longrightarrow \mathbb{R}$, we say that $X$ is a $\mathbb{P}$-martingale if: (i) for each $p \in \mathcal{P}(M), t \leadsto X(t, p)$ is continuous; (ii) for each $t \in[0, \infty), p \leadsto X(t, p)$ is $\mathcal{B}_{t} \equiv \sigma(\{p(\tau): \tau \in[0, t]\})$ measurable and $\mathbb{P}$-integrable, (iii) for each $s<t, X(s)$ is $\mathbb{P}$-almost surely equal to the conditional expectation value $\mathbb{E}^{\mathbb{P}}\left[X(t) \mid \mathcal{B}_{s}\right]$ of $X(t)$ given $\mathcal{B}_{s}$.

${ }^{5}$ We use $\mathbf{M}_{1}(\mathcal{P}(M))$ to denote the space of probability measures on $\mathcal{P}(M)$; and weak convergence on $\mathrm{M}_{1}((\mathcal{M}))$ means convergence when tested with bounded, continuous functions. See, for example, $\S 3.1$ in [12].

${ }^{6} \mathrm{~A}[0, \infty]$-valued function on $\mathcal{P}(M)$ is called a stopping time if, for each $t \in[0, \infty)$, the level set $\{\zeta \leq t\}$ is $\mathcal{B}_{t}$-measurable. Given a stopping time $\zeta$, the $\sigma$-algebra $\mathcal{B}_{\zeta}$ is the collection of $A \subseteq \mathcal{P}(M)$ such that $A \cap\{\zeta \leq t\} \in \mathcal{B}_{t}$ for all $t \in[0, \infty)$. For more details, see $\$ 7.1$ in $[12]$.
} 
Chapter 7, especially Theorem 7.2.3, in [15]), no problem is presented by the uniqueness assertion in the first part of the statement. In addition, the accompanying existence statement comes down to checking that (cf. $\S 2.1$ in [13]) explosion never occurs; and, in conjunction with uniqueness and (1.5), the asserted continuity will follow from the sort of locally uniform non-explosion estimate given by

$$
\lim _{R \rightarrow \infty} \sup _{x \in K} \mathbb{P}_{x}^{U}\left(\sup _{t \in[0, T]} \operatorname{dist}(p(t), x) \geq R\right)=0
$$

for all $T>0$ and compact $K \subseteq M$. Hence, we will turn our attention to proving $(1.5),(1.9)$, and the properties claimed in the second part of the theorem.

We begin by remarking that when $U \equiv 0$ we are dealing with the Brownian motion on $M$, and, because of our assumption that the Ricci curvature is uniformly bounded below, neither existence nor (1.9) causes any problems (cf. Theorem 8.62 in [13], and take into account that the quantity $\mathbb{P}_{x}^{0}$ here is denoted by $\mathbb{P}_{x}^{M}$ there). Moreover, the reversibility statement follows (cf. Exercise 7.5.44 in [12]) in this case from the symmetry property given in (8.36) of [13] (where $\mathbf{P}_{t}^{M}$ there is used to denote what the quantity $\mathbf{P}_{t}^{0}$ will, starting in $\S 2$, stand for here). Finally, it will be important to remember (cf. Theorem 6.25 in [13]) that, for any Borel measurable $\varphi: M \longrightarrow[0, \infty)$, (cf. $(0.3)$ )

$$
\mathbb{E}^{\mathbb{P}_{x}^{0}}[\varphi(p(t))]=\int_{M} \varphi(y) p_{t}^{0}(x, y) \lambda_{M}(d y), \quad(t, x) \in(0, \infty) \times M
$$

Next assume that $U \in C_{\mathrm{c}}^{\infty}(M ;[0, \infty))$. By any one of a myriad procedures (e.g., the one in $\S 7.5$ in [12], where the derivation is based on the Feynman-Kac formula), it is quite easy to verify that, for each $x \in M$, the one and only choice of $\mathbb{P}_{x}^{U}$ satisfies

$$
\mathbb{E}^{\mathbb{P}_{x}^{U}}[F(p)]=\mathbb{E}^{\mathbb{P}_{x}^{0}}\left[R^{U}(\zeta, p) F(p)\right]
$$

where

$R^{U}(t, p) \equiv \exp \left(\frac{U(p(0))-U(p(t))}{2}+\int_{0}^{t}\left(\frac{1}{2} \Delta U-\frac{1}{4}\|\operatorname{grad} U\|^{2}\right)(p(\tau)) d \tau\right)$

for every bounded stopping time $\zeta$ and every $\mathcal{B}_{\zeta}$-measurable

$$
F: \mathcal{P}(M) \longrightarrow[0, \infty)
$$


Hence, in view of the preceding comments about $\left\{\mathbb{P}_{x}^{0}: x \in M\right\},(1.11)$ completes the case when $U \in C_{\mathrm{c}}^{\infty}(M ;[0, \infty))$.

To get away from the hypothesis that $U \in C_{\mathrm{c}}^{\infty}(M ;[0, \infty))$, we will need two more preparatory observations. The first of these is the estimate (cf. (1.1))

$$
\mathbb{P}_{x}^{U}\left(\sup _{t \in[0, T]} U(p(t)) \geq R\right) \leq R^{-1} e^{C T}(2+U(x)),
$$

which we can prove for $U \in C_{\mathrm{c}}^{\infty}(M ;[0, \infty))$ by the following standard procedure. Namely, because

$$
U(p(t))-\int_{0}^{t}\left(\Delta U-\|\operatorname{grad} U\|^{2}\right)(p(\tau)) d \tau
$$

is a $\mathbb{P}_{x}^{U}$-martingale, Doob's Stopping Time Theorem (cf. Corollary 7.1.15 in [12]) says that

$$
U\left(p\left(t \wedge \zeta_{R}\right)\right)-\int_{0}^{t \wedge \zeta_{R}}\left(\Delta U-\|\operatorname{grad} U\|^{2}\right)(p(\tau)) d \tau
$$

where $\zeta_{R}(p)=\inf \{t \geq 0: U(p(t)) \geq R\}$, is also. In particular, this, together with (1.1), means that

$$
\mathbb{E}^{\mathbb{P}_{x}^{U}}\left[2+U\left(p\left(t \wedge \zeta_{R}\right)\right)\right] \leq 2+U(x)+C \int_{0}^{t} \mathbb{E}^{\mathbb{P}} x\left[2+U\left(p\left(\tau \wedge \zeta_{R}\right)\right)\right] d \tau
$$

or, equivalently, that

$$
\mathbb{E}^{\mathbb{P}} x\left[2+U\left(p\left(t \wedge \zeta_{R}\right)\right)\right] \leq e^{C t}(2+U(x)) .
$$

Finally, take $t=T$ in the preceding, note that

$$
R \mathbb{P}_{x}^{U}\left(\zeta_{R} \leq T\right) \leq \mathbb{E}^{\mathbb{P}}{ }_{x}\left[U\left(p\left(T \wedge \zeta_{R}\right)\right)\right]
$$

and arrive at (1.12).

The second observation which we need is such a mild variation on Lemma 2.11 in [14] that we will simply omit its proof.

Lemma 1.13. Let $U$ be a function of the sort described in the first paragraph of this section, and choose a reference point $o \in M$. Then there exists a sequence $\left\{U_{n}\right\}_{1}^{\infty} \subseteq C_{\mathrm{c}}^{\infty}(M ;[0, \infty))$ such that 
(a) For each $n \geq 1,\left|U-U_{n}\right| \leq \frac{1}{n}$ on $\bar{B}(n) \equiv\{x \in M$ : $\operatorname{dist}(x, o) \leq n\}$.

(b) For each $n \geq 1$,

$$
\sup _{x \in \bar{B}(n)}\left\|\operatorname{grad}_{x} U_{n}\right\| \leq n^{-1}+\sup _{x \in \bar{B}(n+1)}\left\|\operatorname{grad}_{x} U\right\| .
$$

Furthermore, $\operatorname{grad} U_{n} \longrightarrow \operatorname{grad} U$ pointwise on $D(U)$ whereas $\left\|\operatorname{grad} U_{n}\right\| \longrightarrow\|\operatorname{grad} U\|$ uniformly on compacts.

(c) For each $n \geq 1$,

$$
\Delta U_{n} \leq \frac{(n+1) C}{n}\left(1+U_{n}+\left\|\operatorname{grad} U_{n}\right\|\right) \quad \text { on } \bar{B}(n)
$$

Now let a general $U$ be given, and choose $\left\{U_{n}\right\}_{1}^{\infty}$ accordingly, as in Lemma 1.13. Our proof that $\mathbb{P}_{x}^{U}$ exists and has the desired properties will be based on our showing that $\left\{\mathbb{P}_{x}^{U_{n}}: n \geq 1\right\}$ is relatively compact in the weak topology on $\mathbf{M}_{1}(\mathcal{P}(M))$ and that every limit point satisfies the conditions required of $\mathbb{P}_{x}^{U}$. To prove the relative compactness, set $\zeta_{R}(p)=\inf \{t \geq 0: U(p(t)) \geq R\}$, and conclude from (1.1) and (1.11) that, for each $(T, R) \in(0, \infty)^{2}$ there is $C(T, R)<\infty$ such that $^{7}$

$$
\sup _{n \geq 1} \mathbb{E}^{\mathbb{P}_{x}^{U_{n}}}\left[F(p), \zeta_{R}(p)>T\right] \leq C(T, R) \mathbb{E}^{\mathbb{P}_{x}^{0}}\left[F(p), \zeta_{R}(p)>T\right]
$$

for all $\mathcal{B}_{T}$-measurable $F: \mathcal{P}(M) \longrightarrow[0, \infty)$. In fact, when $U$ is bounded, $C(T, R)$ can be taken to be independent of $R$ and therefore the desired compactness is immediate. On the other hand, when $U$ is unbounded, we can use (1.12) to see that

$$
\lim _{R \rightarrow \infty} \sup _{n \geq 1} \mathbb{E}^{\mathbb{P}_{x}^{U_{n}}}\left[F(p), \zeta_{R}(p) \leq T\right]=0
$$

Hence, in this case also, $\left\{\mathbb{P}_{x}^{U_{n}}: n \geq 1\right\}$ is relatively compact. Furthermore, if $\mathbb{P}$ is any limit point, then it is clear that

$$
\mathbb{E}^{\mathbb{P}}\left[F(p), \zeta_{R}(p)>T\right] \leq C(R, T) \mathbb{E}^{\mathbb{P}_{x}^{0}}\left[F(p), \zeta_{R}(p)>T\right]
$$

for all non-negative, $\mathcal{B}_{T}$-measurable $F$ 's. In particular, because of (1.10), this means that, for any $\varphi \in C_{\mathrm{c}}^{\infty}(M ; \mathbb{R})$,

$$
\begin{aligned}
& \mathbb{E}^{\mathbb{P}}\left[\int_{0}^{T}\left|L^{U} \varphi-L^{U_{n}} \varphi\right|(p(t)) d t, \zeta_{R}(p)>T\right] \\
& \quad \leq C(T, R) \int_{0}^{T}\left|L^{U} \varphi-L^{U_{n}} \varphi\right|(y) p_{t}^{0}(x, y) \lambda_{M}(d y) \longrightarrow 0
\end{aligned}
$$

\footnotetext{
${ }^{7}$ We use $\mathbb{E}^{\mathbb{P}}[F, A]$ to denote $\mathbb{E}^{\mathbb{P}}\left[F \mathbf{1}_{A}\right]$.
} 
as $n \rightarrow \infty$. Hence, after combining this with the preceding, we can easily check that the martingale characterization for the $\mathbb{P}_{x}^{U_{n}}$ 's becomes the required martingale property for $\mathbb{P}$. That is, $\mathbb{P}=\mathbb{P}_{x}^{U}$; and so $\mathbb{P}_{x}^{U}$ not only exists but is also the limit as $n \rightarrow \infty$ of $\left\{\mathbb{P}_{x}^{U_{n}}: n \geq 1\right\}$. In addition, it is clear that (1.12) continues to hold for $\mathbb{P}_{x}^{\stackrel{x}{U}}$ and that (1.11) leads to the estimate in (1.7); and, of course, (1.5) follows from (1.7), (1.10) and $\lambda_{M}(D(U) C)=0$. Thus, all that remains is to verify the reversibility assertion. But when $F$ is a bounded, continuous $\mathcal{B}_{T}$ measurable function which vanishes whenever either $p(0)$ or $p(T)$ lies outside

a some fixed compact subset of $M, \mathbb{E}^{\mathbb{P}_{n}}[F(p)] \longrightarrow \mathbb{E}^{\mathbb{P}^{U}}[F(p)]$. Thus the reversibility for general $U$ 's follows from reversibility for smooth, compactly supported $U$ 's.

\section{The basic estimate}

Let $U$ be a function of the sort described at the beginning of $\S 1$, and determine the family $\left\{\mathbb{P}_{x}^{U}: x \in M\right\}$ accordingly, as in Theorem 1.4. As a consequence (cf. Theorem 6.22 in [15]) of uniqueness, one knows that this family is Markov in the sense that, for any stopping time $\zeta$ and Borel measurable $F: \mathcal{P}(M) \longrightarrow[0, \infty)$,

$$
\mathbb{E}^{\mathbb{P}_{x}^{U}}\left[F \circ \Sigma_{\zeta} \mid \mathcal{B}_{\zeta}\right](p)=\mathbb{E}^{\mathbb{P}_{p(\zeta)}^{U}}[F] \quad \mathbb{P}_{x}^{U} \text {-almost surely on }\{\zeta<\infty\},
$$

where $\Sigma_{\zeta}: \mathcal{P}(M) \longrightarrow \mathcal{P}(M)$ is the shift map given by

$$
\left[\Sigma_{\zeta}(p)\right](t)= \begin{cases}p(t+\zeta(p)) & \text { if } \zeta(p)<\infty \\ p(t) & \text { if } \zeta(p)=\infty\end{cases}
$$

In particular, if, for $t>0, \mathbf{P}_{t}^{U}$ is the operator determined by

$$
\mathbf{P}_{t}^{U} \varphi(x) \equiv \mathbb{E}_{t}^{\mathbb{P}} \stackrel{U}{x}[\varphi(p(t))]
$$

when $\varphi: M \longrightarrow \mathbb{R}$ is Borel measurable and bounded below, then $\left\{\mathbf{P}_{t}^{U}\right.$ : $t>0\}$ forms a Markov (i.e., non-negativity preserving) semigroup for which $\mathbf{1}$ is invariant. Furthermore, as a consequence of reversibility, it is clear that $\mathbf{P}_{t}^{U}$ is symmetric with respect to $m^{U}$ in the sense that

$$
\int_{M} \varphi \mathbf{P}_{t}^{U} \psi d m^{U}=\int_{M} \psi \mathbf{P}_{t}^{U} \varphi d m^{U}
$$


for all non-negative, Borel measurable $\varphi$ 's. In particular, this leads, via Jensen's inequality, to the conclusion that, for each $q \in[1, \infty]$ and $t>0$, $\mathbf{P}_{t}^{U}$ is a contraction in $L^{q}\left(m^{U} ; \mathbb{R}\right)$ :

$$
\left\|\mathbf{P}_{t}^{U} \varphi\right\|_{L^{q}\left(m^{U} ; \mathbb{R}\right)} \leq\|\varphi\|_{L^{q}\left(m^{U} ; \mathbb{R}\right)^{*}} .
$$

Of course, the reversibility can be combined with the contractivity to show that each $\mathbf{P}_{t}^{U}$ admits a unique extension $\overline{\mathbf{P}_{t}^{U}}$ as a self-adjoint contraction on $L^{2}\left(m^{U} ; \mathbb{R}\right)$, and clearly $\left\{\overline{\mathbf{P}_{t}^{U}}: t>0\right\}$ is a strongly continuous semigroup of self-adjoint Markov operators on $L^{2}\left(m^{U} ; \mathbb{R}\right)$. In fact, if

$$
\mathcal{E}^{U}(\varphi, \varphi) \equiv \lim _{t \searrow 0} \frac{1}{t}\left(\varphi, \varphi-\overline{\mathbf{P}_{t}^{U}} \varphi\right)_{L^{2}\left(m^{U} ; \mathbb{R}\right)}, \quad \varphi \in L^{2}\left(m^{U} ; \mathbb{R}\right),
$$

is the Dirichlet form associated with this semigroup, then

$$
\mathcal{E}^{U}(\varphi, \varphi)=\int_{M}\|\operatorname{grad} \varphi\|^{2} d m^{U} \quad \text { for } \varphi \in C_{c}^{1}(M ; \mathbb{R})
$$

To prove (2.6), one simply has to remember that

$$
\mathbf{P}_{t}^{U} \varphi-\varphi=\int_{0}^{t} \mathbf{P}_{\tau}^{U} L^{U} \varphi d \tau, \quad \varphi \in C_{\mathrm{c}}^{\infty}(M ; \mathbb{R})
$$

and then apply integration by parts and an easy approximation argument.

In order to state the main result of this section, let $o \in M$ be a fixed reference point, use $\mathcal{C}$ to denote the cut-locus of $o$, and set

$$
\rho(x)=\operatorname{dist}(x, o) \quad \text { for } x \in M \text {. }
$$

Next, for each $\epsilon \in(0,1)$, let $\mathcal{U}_{\epsilon}$ denote the set of $U: M \longrightarrow[0, \infty)$ satisfying, in addition to the conditions introduced at the beginning of $\S 1,8$

$$
e^{-\lambda U} \Delta e^{\lambda U} \leq \lambda^{2}\|\operatorname{grad} U\|^{2}+C \lambda(1+U+\|\operatorname{grad} U\|)
$$

in the sense of distributions for all $\lambda>0$ and some $C<\infty, \epsilon U^{1+\epsilon} \leq$ $1+\|\operatorname{grad} U\|^{2}$, and $\epsilon \rho^{1+\epsilon} \leq 1+U$.

\footnotetext{
${ }^{8}$ It should be noted that the first part of the following is the same as the condition in (1.1) when the function $U$ is twice differentiable.
} 
Example. The basic example from which this definition of $\mathcal{U}_{\epsilon}$ was abstracted is the one when $U=f \circ \rho$ where $f:[0, \infty) \longrightarrow[0, \infty)$ is a twice continuously differentiable which satisfies the conditions

$$
\begin{gathered}
f(0)=f^{\prime}(0)=0, \quad f(1)=1, \quad r f^{\prime}(r) \geq \frac{2}{1-\epsilon} f(r), r>0, \\
\quad \text { and } C_{f} \equiv \sup _{r>0} \frac{f^{\prime \prime}(r)}{1+f(r)}<\infty .
\end{gathered}
$$

To see that this choice of $U$ is an element of $\mathcal{U}_{\epsilon}$, let $\mathcal{C}$ denote the cutlocus of $o$, and recall that $\{o\} \cup \mathcal{C}$ is a closed set of $\lambda_{M}$-measure 0 off of which $\rho$ is a smooth function satisfying $\|\operatorname{grad} \rho\|=1$. In addition, by $(2.9)$, it is easy to check that

$$
\begin{gathered}
\rho \leq 1 \Longrightarrow f \circ \rho \leq 1, \quad \rho \geq 1 \Longrightarrow f \circ \rho \geq \rho^{\frac{2}{1-\epsilon}}, \\
\text { and } C_{f} \rho(1+f \circ \rho) \geq\|\operatorname{grad} f \circ \rho\| \geq \frac{2 f \circ \rho}{(1-\epsilon) \rho} \geq \frac{2}{1-\epsilon}(f \circ \rho)^{\frac{1+\epsilon}{2}},
\end{gathered}
$$

from which it is clear that conditions in the second line of (2.8) are met. Finally, by a slight extension (cf. (5.39) in [13]) of an estimate initiated by E. Calabi in [4] and improved by S-T Yau in [19],

$$
\Delta g \circ \rho \leq g^{\prime \prime} \circ \rho+\left(\frac{d-1}{\rho}+\sqrt{d \kappa_{\text {Ric }}}\right) g^{\prime} \circ \rho
$$

in the sense of distributions for any twice continuously differentiable, non-decreasing function $g$ on $[0, \infty)$ whose first derivative vanishes at 0 . Hence the first line of (2.8) follows when one applies this to $g=e^{\lambda f}$ and takes into account the size conditions imposed on $f^{\prime \prime}$.

Our goal in this section is to prove the following theorem, which is the analytic engine which drives everything else in this article.

Theorem 2.11. Let $U: M \longrightarrow[0, \infty)$ satisfy the conditions stated at the beginning of $\S 1$. If there exists an $\epsilon>0$ and $a \tilde{U} \in \mathcal{U}_{\epsilon}$ for which $\|U-\tilde{U}\|_{\mathrm{u}}<\infty$, then there exists a $\nu_{U} \in[0, \infty)$ such that

$$
\left\|\mathbf{P}_{t}^{U} \varphi\right\|_{L^{2}\left(m^{U}\right)} \leq A_{U} \exp \left(A_{U} t^{-\nu_{U}}\right)\|\varphi\|_{L^{1}\left(m^{U}\right)}, \quad t \in(0, \infty),
$$

for all $\varphi \in C_{\mathrm{b}}(M ; \mathbb{R})$.

Because of a beautiful observation made by $\mathrm{T}$. Coulhon in [5], it suffices to prove the estimate in (2.12) when $U$ itself is an element of $\mathcal{U}_{\epsilon}$. More precisely, Coulhon's Theorem II.5 guarantees that if $\tilde{U}$ satisfies an 
estimate of the form in (2.12), then so will any other $U$ whose associated Dirichlet form is commensurate with the Dirichlet form determined by $\tilde{U}$.

With the preceding remark in mind, we devote the rest of this section to the derivation of (2.12) when $U \in \mathcal{U}_{\epsilon}$, and the first step is to notice that, because $\mathbf{P}_{t}^{U}$ is symmetric on $L^{2}\left(m^{U}\right)$, an easy duality argument shows that (2.12) is equivalent (with a different choice of $A_{U}$ ) to

$$
\begin{array}{r}
\left\|\mathbf{P}_{t}^{U} \varphi\right\|_{\mathrm{u}} \leq A_{U} \exp \left(A_{U} t^{-\nu_{U}}\right)\|\varphi\|_{L^{2}\left(m^{U}\right)} \\
\text { for all } t \in(0, \infty) \text { and } \varphi \in C_{\mathrm{b}}(M ; \mathbb{R})
\end{array}
$$

The next step is to show that there exists a $C^{\prime}<\infty$ such that

$$
\left|\mathbf{P}_{T}^{U} \varphi(x)\right| \leq C^{\prime} t^{-\frac{\operatorname{dim}(M)}{4}} \exp \left(\frac{3}{4} U(x)\right)\|\varphi\|_{L^{2}\left(m^{U}\right)}, \quad T \in(0,1],
$$

for any $\varphi \in C_{\mathrm{b}}(M ; \mathbb{R})$. But, by plugging the estimates in (2.8) into (1.6) and then using (1.7), we can find a $C^{\prime \prime}<\infty$ for which

$$
\left|\mathbf{P}_{T}^{U} \varphi(x)\right| \leq C^{\prime \prime} e^{\frac{1}{2} U(x)} \mathbb{E}^{\mathbb{P}_{x}^{0}}\left[\left(e^{-\frac{1}{2} U}|\varphi|\right)(p(T))\right], \quad T \in(0,1] .
$$

At the same time, (cf. (0.3))

$$
\begin{aligned}
\left|\mathbb{E}_{t}^{\mathbb{P} 0}\left[\left(e^{-\frac{1}{2} U} \varphi\right)(p(T))\right]\right| & \leq\left[\mathbf{P}_{T}^{0}\left(e^{-\frac{1}{2} U}|\varphi|\right)\right](x) \\
& =\int_{M}\left(e^{-\frac{1}{2} U}|\varphi|\right)(y) p_{T}^{0}(x, y) \lambda_{M}(d y) \\
& \leq\left\|p_{T}^{0}(x, \cdot)\right\|_{L^{2}\left(\lambda_{M}\right)}\|\varphi\|_{L^{2}\left(m^{U}\right)} .
\end{aligned}
$$

To complete the proof of (2.14) from here, we use the symmetry of $p_{t}^{0}(x, y)$ in and $x$ and $y$ together with the Chapman-Kolmogorov equation to see that $p_{2 T}^{0}(x, x)=\left\|p_{T}^{0}(x, \cdot)\right\|_{L^{2}\left(\lambda_{M}\right)}^{2}$ and therefore that

$$
\left|\mathbf{P}_{T}^{U} \varphi(x)\right| \leq C^{\prime \prime} e^{\frac{1}{2} U(x)} \sqrt{p_{2 T}^{0}(x, x)}\|\varphi\|_{L^{2}\left(m^{U}\right)} .
$$

Finally, after combining the estimate of Li and Yau (cf. Corollary 3.1 in [9]) with Bishop's volume comparison theorem, we find that

$$
p_{2 T}^{0}(x, x) \leq K^{2} T^{-\frac{\operatorname{dim}(M)}{2}} e^{2 K \rho(x)}, \quad(T, x) \in(0,1] \times M \times M,
$$

for some $K \in(0, \infty)$ depending only on $\operatorname{dim}(M)$ and $\kappa_{\text {Ric }}$, and obviously (cf. (2.8)) this finishes the derivation of (2.14). 
Given (2.14), what we still need to do is estimate the action of $\mathbf{P}_{t}^{U}$ on the right hand side of (2.14). Indeed, once we can get the right sort of estimate, then we will be able to get (2.13) by using writing $\mathbf{P}_{2 T}^{U}=\mathbf{P}_{T}^{U} \circ \mathbf{P}_{T}^{U}$ and using (2.14). The estimate which we seek will be obtained via a comparison argument, which rests on the following somewhat delicate form of the weak maximum principle.

Lemma 2.15. Suppose that $u:[0, T] \times M \longrightarrow[0, \infty)$ is a continuous function which is continuously differentiable on $[0, T]$ for each $x \in M$ and locally Lipschitz continuous on $M$ for each $t \in[0, T]$. Further, assume that $\dot{u}=\frac{\partial u}{\partial t}$ is continuous on $[0, T] \times M$ and that

$$
\int_{0}^{T} \int_{M}(|\dot{u}|+\|\operatorname{grad} u\|) d t d m^{U}<\infty
$$

If $L^{U} u(t) \leq \dot{u}(t)$ in the sense that

$$
\int_{M}\left(L^{U} \varphi\right) u(t) d m^{U} \leq \int_{M} \varphi \dot{u}(t) d m^{U}
$$

for all $\varphi \in C_{\mathrm{c}}^{\infty}(M ;[0, \infty))$ and $t \in[0, T]$, then

$$
\mathbf{P}_{T}^{U} u(0) \leq u(T) \quad \text { on } M
$$

Proof. We can (e.g., see Theorem 4.2 in [16], which does much more) find a smooth function $\delta$ whose gradient is uniformly bounded and whose level sets are compact. Next, choose $\psi \in C^{\infty}(\mathbb{R} ;[0,1])$ so that $\psi \equiv 1$ on $[-1,1]$ and $\psi \equiv 0$ off $[-2,2]$, and set $\psi_{R}(x)=\psi\left(R^{-1} \delta(x)\right)$ for $R>0$ and $x \in M$. Finally, referring to Lemma 1.13, define

$$
L^{(n, R)} \varphi=e^{U_{n}} \operatorname{div}\left(\psi_{R} e^{-U_{n}} \operatorname{grad} \varphi\right) \quad \text { for } \varphi \in C^{2}(M ; \mathbb{R}) .
$$

Because all the coefficients in $L^{(n, R)}$ are smooth and compactly supported, there is no problem about constructing a Markov semigroup $\left\{\mathbf{P}_{t}^{(n, R)}: t>0\right\}$ on $C_{\mathrm{b}}(M ; \mathbb{R})$ such that

$$
\mathbf{P}_{t}^{(n, R)} \varphi-\varphi=\int_{0}^{t} \mathbf{P}_{\tau}^{(n, R)} L^{(n, R)} \varphi d \tau, \quad \varphi \in C_{\mathrm{c}}^{2}(M ; \mathbb{R})
$$

In fact, if $V_{R} \equiv\{x \in M: \delta(x)<R\}$, then $C_{\mathrm{C}}^{\infty}\left(V_{2 R} ; \mathbb{R}\right)$ is $\left\{\mathbf{P}_{t}^{(n, R)}: t>0\right\}$-invariant and

$$
\frac{d}{d t} \mathbf{P}_{t}^{(n, R)} \varphi=L^{(n, R)} \mathbf{P}_{t}^{(n, R)} \varphi, \quad \varphi \in C_{\mathrm{c}}^{\infty}\left(V_{2 R} ; M\right) .
$$


Furthermore, each $\mathbf{P}_{t}^{(n, R)}$ is symmetric with respect to $m^{U_{n}}$, and the Dirichlet form associated with the corresponding semigroup of selfadjoint contractions is given by

$$
\int_{M} \psi_{R}\|\operatorname{grad} \varphi\|^{2} d m^{U_{n}} \quad \text { for } \varphi \in C_{\mathrm{c}}^{\infty}\left(V_{2 R} ; \mathbb{R}\right)
$$

Thus, elementary spectral theory considerations lead to the conclusion that

$$
\int_{M} \psi_{R}\left\|\operatorname{grad} \mathbf{P}_{t}^{(n, R)} \varphi\right\|^{2} d m^{U_{n}} \leq \int_{M} \psi_{R}\|\operatorname{grad} \varphi\|^{2} d m^{U_{n}}, \quad t>0
$$

for $\varphi \in C_{\mathrm{c}}^{\infty}\left(V_{2 R} ; \mathbb{R}\right)$. Finally, for each $R>0$ and $\varphi \in C_{\mathrm{b}}(M ; \mathbb{R})$,

$$
\mathbf{P}_{t}^{(n, R)} \varphi(x) \longrightarrow \mathbf{P}_{t}^{(R)} \varphi(x) \quad \text { uniformly on compacts }
$$

as $n \rightarrow \infty$, where $\left\{\mathbf{P}_{t}^{(R)}: t>0\right\}$ is the Markov semigroup which satisfies

$$
\mathbf{P}_{t}^{(R)} \varphi-\varphi=\int_{0}^{t} \mathbf{P}_{\tau}^{(R)} L^{(R)} \varphi d \tau, \quad \varphi \in C_{\mathrm{c}}^{2}(M ; \mathbb{R})
$$

with $L^{(R)} \varphi \equiv e^{U} \operatorname{div}\left(\psi_{R} e^{-U} \operatorname{grad} \varphi\right)$, and we have used the subterfuge introduced in (1.2) off of $D(U)$. In particular, for each $\varphi \in C_{\mathrm{b}}(M ; \mathbb{R})$, $\mathbf{P}_{t}^{(R)} \varphi \longrightarrow \mathbf{P}_{t}^{U} \varphi$ uniformly on compacts as $R \rightarrow \infty$.

With these preliminaries, we can now prove the result as follows. Let $\varphi \in C_{\mathrm{c}}^{\infty}(M ;[0, \infty))$ be given, and suppose $R$ is taken so that the support of $\varphi$ is contained in $V_{R}$. Then

$$
\begin{aligned}
& \frac{d}{d t} \int_{M}\left(\mathbf{P}_{t}^{(n, R)} \varphi\right) u(T-t) d m^{U} \\
& \quad=\int_{M}\left(\left(L^{(n, R)} \mathbf{P}_{t}^{(n, R)} \varphi\right) u(T-t)-\left(\mathbf{P}_{t}^{(n, R)} \varphi\right) \dot{u}(T-t)\right) d m^{U}
\end{aligned}
$$

Next, observe that

$$
L^{(n, R)} \varphi=L^{U_{n}}\left(\psi_{R} \varphi\right)-e^{U_{n}} \operatorname{div}\left(e^{-U_{n}} \varphi \operatorname{grad} \psi_{R}\right)
$$

and conclude that the right hand side of the preceding can be re-written 
as

$$
\begin{aligned}
& \int_{M}\left(L^{U}\left(\psi_{R} \mathbf{P}_{t}^{(n, R)} \varphi\right) u(T-t)-\left(\psi_{R} \mathbf{P}_{t}^{(n, R)} \varphi\right) \dot{u}(T-t)\right) d m^{U} \\
& \quad+\int_{M}\left\langle\operatorname{grad}\left(U-U_{n}\right), \operatorname{grad}\left(\psi_{R} \mathbf{P}_{t}^{(n, R)} \varphi\right)\right\rangle u(T-t) d m^{U} \\
& \quad-\int_{M}\left(1-\psi_{R}\right)\left(\mathbf{P}_{t}^{(n, R)} \varphi\right) \dot{u}(T-t) d m^{U} \\
& \quad+\int_{M}\left\langle\operatorname{grad} \psi_{R}, \operatorname{grad}\left(e^{U_{n}-U} u(T-t)\right)\right\rangle \mathbf{P}_{t}^{(n, R)} \varphi d m^{U_{n}} \\
& \leq \int_{M}\left\langle\operatorname{grad}\left(U-U_{n}\right), \operatorname{grad} \mathbf{P}_{t}^{(n, R))} \varphi\right\rangle \psi_{R} u(T-t) d m^{U} \\
& \quad+\int_{M}\left\langle\operatorname{grad} \psi_{R}, \operatorname{grad} u(T-t)\right\rangle \mathbf{P}_{t}^{(n, R)} \varphi d m^{U} \\
& \quad-\int_{M}\left(1-\psi_{R}\right)\left(\mathbf{P}_{t}^{(n, R)} \varphi\right) \dot{u}(T-t) d m^{U} .
\end{aligned}
$$

Hence, after integrating over $[0, T]$, we find that

$$
\begin{aligned}
\int_{M} \mathbf{P}_{T}^{(n, R)} \varphi u(0) d m^{U}-\int_{M} \varphi u(T) d m^{U} \\
\leq \int_{0}^{T}\left(\int_{M}\left\langle\operatorname{grad}\left(U-U_{n}\right), \operatorname{grad} \mathbf{P}_{t}^{(n, R))} \varphi\right\rangle \psi_{R} u(T-t) d m^{U}\right) d t \\
\quad+\int_{0}^{T}\left(\int_{M}\left\langle\operatorname{grad} \psi_{R}, \operatorname{grad} u(T-t)\right\rangle \mathbf{P}_{t}^{(n, R)} \varphi d m^{U}\right) d t \\
\quad-\int_{0}^{T}\left(\int_{M}\left(1-\psi_{R}\right) \mathbf{P}_{t}^{(n, R)} \varphi \dot{u}(T-t) d m^{U}\right) d t
\end{aligned}
$$

Finally, by first letting $n \rightarrow \infty$ and using (2.17), and then letting $R \rightarrow \infty$ and using the integrability hypotheses about $u$ and its gradient, we conclude that

$$
\int_{M}\left(\mathbf{P}_{T}^{U} \varphi\right) u(0) d m^{U} \leq \int_{M} \varphi u(T) d m^{U},
$$

from which $\mathbf{P}_{T}^{U} u(0) \leq u(T)$ follows immediately after applying (2.3).

q.e.d.

We now want to use Lemma 2.15 to prove that there exists an $A \in$ $[1, \infty)$ such that $($ cf. $(2.8))$

$$
\left\|\mathbf{P}_{T}^{U}\left(e^{\frac{3}{4} U}\right)\right\|_{\mathrm{u}} \leq A T^{-\frac{d}{4}} \exp \left(A T^{-\frac{1}{\epsilon}}\right), \quad T \in(0,1] .
$$


For this purpose, we seek a comparison function $u$ of the form

$$
u(t, x)=\exp (\lambda(t) U(x)+\mu(t)),
$$

where $\lambda$ and $\mu$ are non-negative, continuously differentiable, monotonic functions on $[0, T]$ with $\lambda(0)=\frac{3}{4}$ and $\lambda(T)=0=\mu(0)$. Notice that, by (2.8), we have, in the sense of distributions, the inequality

$$
L^{U} u(t) \leq \lambda(t)\left[B(1+U+\|\operatorname{grad} U\|)-(1-\lambda(t))\|\operatorname{grad} U\|^{2}\right] u(t),
$$

for some $B \in[1, \infty)$. Hence, in order to assure that $L^{U} u(t) \leq \dot{u}(t)$, in the sense of $(2.16)$, we need only require that

$$
\left(B-\frac{\dot{\lambda}(t)}{\lambda(t)}\right) U+B(1+\|\operatorname{grad} U\|)-(1-\lambda(t))\|\operatorname{grad} U\|^{2} \leq \frac{\dot{\mu}(t)}{\lambda(t)}
$$

which, because $\lambda(t) \leq \frac{3}{4}$ and $1+\|\operatorname{grad} U\|^{2} \geq \epsilon U^{1+\epsilon}$, will hold if

$$
\left(1+3 B^{2}\right)+\left(B-\frac{\dot{\lambda}(t)}{\lambda(t)}\right) U-\frac{\epsilon}{8} U^{1+\epsilon} \leq \frac{\dot{\mu}(t)}{\lambda(t)}
$$

Now notice that, for any $\xi \geq 0$, (cf. (2.18))

$$
\sup \left\{1+3 B^{2}+\xi \sigma-\frac{\epsilon}{8} \sigma^{1+\frac{1}{\epsilon}}: \sigma>0\right\} \leq B^{\prime}(1+\xi)^{1+\frac{1}{\epsilon}}
$$

for some $B^{\prime} \in[1, \infty)$, and conclude that we can achieve our goal by taking

$$
\mu(t)=B^{\prime} \int_{0}^{t} \lambda(\tau)\left(1+B-\frac{\dot{\lambda}(\tau)}{\lambda(\tau)}\right)^{1+\frac{1}{\epsilon}} d \tau
$$

That is, by Lemma 2.15, we now know that

$$
\left\|\mathbf{P}_{T}^{U} e^{\frac{3}{4}} U\right\|_{\mathrm{u}} \leq \exp \left[B^{\prime} \int_{0}^{T} \lambda(\tau)\left(1+B^{\prime}-\frac{\dot{\lambda}(\tau)}{\lambda(\tau)}\right)^{1+\frac{1}{\epsilon}} d \tau\right]
$$

for any smooth, decreasing function $\lambda$ which satisfies $\lambda(0)=\frac{3}{4}$ and $\lambda(T)=0$. In particular, by choosing $\lambda(t)=\frac{3}{4}\left(1-\frac{t}{T}\right)^{1+\frac{1}{\epsilon}}$, we arrive at (2.18) for an appropriate choice of $A$. 
To complete the proof of (2.13) from here, first note that, because of (2.4) with $q=2$, it suffices to handle $t \in(0,1]$. Second, given $t \in(0,1]$, take $T=\frac{t}{2}$ in (2.14) and (2.18), and thereby conclude that

$$
\left\|\mathbf{P}_{t}^{U} \varphi\right\|_{\mathbf{u}} \leq 2^{\frac{d}{4}} C^{\prime} t^{-\frac{d}{4}} \exp \left(2^{\frac{1}{\epsilon}} A t^{-\frac{1}{\epsilon}}\right) .
$$

Clearly (2.13) for $t \in(0,1]$ results from this when we take any $\nu_{U}>\frac{1}{\epsilon}$ and then choose $A_{U}$ appropriately.

\section{The Hodge operator and the bundle of orthonormal frames}

For reasons which will become clear in the ensuing sections, it will be convenient to have available the expressions which the familiar exterior differential and related operations take when they are written in terms of the bundle $\mathcal{O}(M)$ of orthonormal frames (cf. [1] or Chapter 8 in [13], which introduces the same notation and conventions as those adopted here). As a first step, recall that if $\omega \in \Omega^{q}(M)$ (i.e., $\omega$ is a smooth $q$-form on $M$ ) and $x \in M$, then, for any coordinate system at $x$,

$$
(d \omega)_{x}\left(\partial_{i_{0}}, \ldots, \partial_{i_{q}}\right)=\sum_{\mu=0}^{q}(-1)^{\mu}\left(\partial_{i_{\mu}}\right)_{x} \omega\left(\partial_{i_{0}}, \ldots, \widehat{\partial_{\mu}}, \ldots, \partial_{i_{q}}\right) .
$$

In particular, if the coordinate system is normal at $x$, and therefore $\left(\partial_{i_{\mu}}\right)_{x} \omega\left(\partial_{i_{0}}, \ldots, \widehat{\partial_{\mu}}, \ldots, \partial_{i_{q}}\right)=\left(\nabla_{\left(\partial_{i_{\mu}}\right) x} \omega\right)\left(\left(\partial_{i_{0}}\right)_{x}, \ldots, \widehat{\left(\partial_{\mu}\right)_{x}}, \ldots,\left(\partial_{i_{q}}\right)_{x}\right)$ we can conclude that, for any vectors $\left(\left(X_{0}\right)_{x}, \ldots,\left(X_{q}\right)_{x}\right) \in\left(T_{x} M\right)^{q}$,

$$
\begin{aligned}
& (d \omega)_{x}\left(\left(X_{0}\right)_{x}, \ldots,\left(X_{q}\right)_{x}\right) \\
& \quad=\sum_{\mu=0}^{q}(-1)^{\mu}\left(\nabla_{\left(X_{\mu}\right)_{x}} \omega\right)\left(\left(X_{0}\right)_{x}, \ldots, \widehat{\left(X_{\mu}\right)_{x}}, \ldots,\left(X_{q}\right)_{x}\right) .
\end{aligned}
$$

Hence, if $\left(\left(X_{1}\right)_{x}, \ldots,\left(X_{d}\right)_{x}\right)$ is a basis in $T_{x} M$ and $\left(\left(\eta^{1}\right)_{x}, \ldots,\left(\eta^{d}\right)_{x}\right)$ is the associated dual basis in $\left(T_{x} M\right)^{*}$ (i.e., $\left.\left(\eta^{i}\right)_{x}\left(\left(X_{j}\right)_{x}\right)=\delta_{j}^{i}\right)$, then

$$
(d \omega)_{x}=\sum_{i=1}^{d}\left(\eta^{i}\right)_{x} \wedge\left(\nabla_{\left(X_{i}\right)_{x}} \omega\right)
$$

Next, given $\omega \in \Omega^{*}(M) \equiv \bigoplus_{q=0}^{\operatorname{dim}(M)} \Omega^{q}(M)$, define the lift

$$
\tilde{\omega}: \mathcal{O}(M) \longrightarrow \Lambda^{*}\left(\left(\mathbb{R}^{d}\right)^{*}\right) \equiv \bigoplus_{q=0}^{\operatorname{dim}(M)} \Lambda^{q}\left(\left(\mathbb{R}^{d}\right)^{*}\right)
$$


of $\omega$ to $\mathcal{O}(M)$ so that, for $\mathfrak{f} \in \mathcal{O}(M), 1 \leq k \leq \operatorname{dim}(M)$, and $\left(\xi_{1}, \ldots, \xi_{d}\right) \in$ $\left(\mathbb{R}^{d}\right)^{q}$

$$
\tilde{\omega}_{\mathfrak{f}}\left(\xi_{1}, \ldots, \xi_{q}\right)=\omega_{\pi \mathfrak{f}}\left(\mathfrak{f} \xi_{1}, \ldots, \mathfrak{f} \xi_{q}\right),
$$

where $\pi: \mathcal{O}(M) \longrightarrow M$ is the fiber map and we have adopted the notation (cf. (8.1) in [13]) $\mathfrak{f} \xi$ to denote the element of $T_{\pi \mathfrak{f}} M$ whose components in the orthonormal frame $f$ are $\xi=\left(\xi^{1}, \ldots, \xi^{d}\right) \in \mathbb{R}^{d}$. Notice that a smooth $\Phi: \mathcal{O}(M) \longrightarrow \Lambda^{*}\left(\left(\mathbb{R}^{d}\right)^{*}\right)$ is the lift of some $\omega \in \Omega^{*}(M)$ if and only if $\Phi$ is rotation equivariant in the sense that, for any orthogonal transformation $O \in \mathcal{O}\left(\mathbb{R}^{d}\right)$,

$$
\Phi_{R_{O} \mathfrak{f}}\left(\xi_{1}, \ldots, \xi_{q}\right)=\Phi_{\mathfrak{f}}\left(O \xi_{1}, \ldots, O \xi_{q}\right)
$$

where (cf. (8.2) in [13]) $R_{O}: \mathcal{O}(M) \longrightarrow \mathcal{O}(M)$ is the map determined so that $R_{O} \mathfrak{f} \xi=\mathfrak{f} O \xi$. The importance to us of lifting forms to $\mathcal{O}(M)$ derives from the fact that

$$
\widetilde{\nabla_{\mathfrak{f} \xi} \omega}=\mathfrak{E}(\xi)_{\mathfrak{f}} \tilde{\omega},
$$

where $\mathfrak{E}(\xi)$ is the canonical vector field (cf. (8.12) in [13]) on $\mathcal{O}(M)$ determined by $\xi \in \mathbb{R}^{d}$. That is, $\mathfrak{E}(\xi)_{\mathfrak{f}}$ is the horizontal lift of $\mathfrak{f} \xi$ to $\mathfrak{f}$. Perhaps the most instructive way to check $(3.3)$ is to let $\mathfrak{p}: \mathbb{R} \longrightarrow \mathcal{O}(M)$ be the integral curve of $\mathfrak{E}(\xi)$ with $\mathfrak{p}(0)=\mathfrak{f}$ and to observe that, for any $\left(\eta_{1}, \ldots, \eta_{q}\right) \in\left(\mathbb{R}^{d}\right)^{q}$

$$
\begin{aligned}
\left(\nabla_{\mathfrak{f} \xi} \omega\right)\left(\eta_{1}, \ldots, \eta_{q}\right) & =\left.\frac{d}{d t} \omega_{\pi \circ \mathfrak{p}(t)}\left(\mathfrak{p}(t) \eta_{1}, \ldots, \mathfrak{p}(t) \eta_{q}\right)\right|_{t=0} \\
& =\left.\frac{d}{d t} \tilde{\omega}_{\mathfrak{p}(t)}\left(\eta_{1}, \ldots, \eta_{q}\right)\right|_{t=0}=(\mathfrak{E}(\xi) \tilde{\mathfrak{f}} \tilde{\omega})\left(\eta_{1}, \ldots, \eta_{q}\right) .
\end{aligned}
$$

In any case, if, for smooth $\Phi: \mathcal{O}(M) \longrightarrow \Lambda^{*}\left(\left(\mathbb{R}^{d}\right)^{*}\right)$, we take

$$
\tilde{d} \Phi \equiv \sum_{i=1}^{d} \mathbf{e}_{i}^{*} \wedge \mathfrak{E}\left(\mathbf{e}_{i}\right) \Phi,
$$

where $\left(\mathbf{e}_{1}, \ldots, \mathbf{e}_{d}\right)$ is any orthonormal basis in $\mathbb{R}^{d}$, then the conjunction of (3.1) with (3.3) leads to

$$
\widetilde{d \omega}=\tilde{d} \tilde{\omega} .
$$

We next want to compute the formal adjoint $\delta$ of $d$ with respect to $\lambda_{M}$. More precisely, given an orthonormal basis $\left(\left(E_{1}\right)_{x}, \ldots,\left(E_{d}\right)_{x}\right)$ in $T_{x} M$ and an

$$
I=\left(i_{1}, \ldots, i_{q}\right) \in(\{1, \ldots, d\})^{q},
$$


set

$$
\left(E_{I}\right)_{x}=\left(\left(E_{i_{1}}\right)_{x}, \ldots,\left(E_{i_{q}}\right)_{x}\right) .
$$

For $\omega_{x} \in \Lambda^{q}\left(\left(T_{x} M\right)^{*}\right)$ and $\omega_{x}^{\prime} \in \Lambda^{q^{\prime}}\left(\left(T_{x} M\right)^{*}\right)$, we take

$$
\left\langle\omega_{x}, \omega_{x}^{\prime}\right\rangle=\frac{1}{q !} \sum_{|I|=q} \omega_{x}\left(\left(E_{I}\right)_{x}\right) \omega_{x}^{\prime}\left(\left(E_{I}\right)_{x}\right)
$$

when $q=q^{\prime}$ and $\left\langle\omega_{x}, \omega_{x}^{\prime}\right\rangle=0$ when $q \neq q^{\prime}$. Then, $\delta$ is to be determined so that

$$
\int_{M}\left\langle\omega, d \omega^{\prime}\right\rangle d \lambda_{M}=\int_{M}\left\langle\delta \omega, \omega^{\prime}\right\rangle d \lambda_{M}
$$

when at least one of $\omega$ or $\omega^{\prime}$ is compactly supported. Equivalently, let $\left(\mathbf{e}_{1}, \ldots, \mathbf{e}_{d}\right)$ be an orthonormal basis in $\mathbb{R}^{d}$, set $\mathbf{e}_{I}=\left(\mathbf{e}_{i_{1}}, \ldots, \mathbf{e}_{i_{q}}\right)$ for $I \in(\{1, \ldots, d\})^{q}$, and, depending on whether $q=q^{\prime}$ or $q \neq q^{\prime}$, set

$$
\left(\Phi, \Phi^{\prime}\right)=\frac{1}{q !} \sum_{|I|=q} \Phi\left(\mathbf{e}_{I}\right) \Phi^{\prime}\left(\mathbf{e}_{I}\right)
$$

or $\left(\Phi, \Phi^{\prime}\right)=0$ for $\Phi \in \Lambda^{q}\left(\left(\mathbb{R}^{d}\right)^{*}\right)$ and $\Phi^{\prime} \in \Lambda^{q^{\prime}}\left(\left(\mathbb{R}^{d}\right)^{*}\right)$. Next, let $\lambda_{\mathcal{O}(M)}$ (cf. $\S 8.1 .4$ in [13]) be the measure on $\mathcal{O}(M)$ whose marginal on $M$ is $\lambda_{M}$ and whose conditional distribution on each fiber is given by the normalized Haar measure $\lambda_{\mathcal{O}\left(\mathbb{R}^{d}\right)}$ on $\mathcal{O}\left(\mathbb{R}^{d}\right)$, and determine the operation $\tilde{\delta}$ so that

$$
\int_{\mathcal{O}(M)}\left(\Phi, \tilde{d} \Phi^{\prime}\right) d \lambda_{\mathcal{O}(M)}=\int_{\mathcal{O}(M)}\left(\tilde{\delta} \Phi, \Phi^{\prime}\right) d \lambda_{\mathcal{O}(M)}
$$

when $\Phi$ and $\Phi^{\prime}$ are smooth maps from $\mathcal{O}(M)$ into $\Lambda^{*}\left(\left(\mathbb{R}^{d}\right)^{*}\right)$, at least one of which is compactly supported. Then $\widetilde{\delta \omega}=\tilde{\delta} \tilde{\omega}$.

To compute $\tilde{\delta} \Phi$, assume $\Phi$ takes its values in $\Lambda^{q}\left(\left(\mathbb{R}^{d}\right)^{*}\right)$. If $q=0$, then we must take $\tilde{\delta} \Phi \equiv 0$. Thus, assume that $1 \leq q \leq \operatorname{dim}(M)$, and let $\Phi^{\prime}: \mathcal{O}(M) \longrightarrow \Lambda^{q-1}\left(\left(\mathbb{R}^{d}\right)^{*}\right)$. Then, because (cf. (8.32) in [13]) $\mathfrak{E}(\xi)$ is formally skew-adjoint with respect to $\lambda_{\mathcal{O}(M)}$, we find from (3.4) that

$$
-\int_{\mathcal{O}(M)}\left(\tilde{\delta} \Phi, \Phi^{\prime}\right) d \lambda_{\mathcal{O}(M)}=\sum_{i=1}^{d} \int_{\mathcal{O}(M)}\left(\mathfrak{E}\left(\mathbf{e}_{i}\right) \Phi, \mathbf{e}_{i}^{*} \wedge \Phi^{\prime}\right) d \lambda_{\mathcal{O}(M)}
$$

Hence, because

$$
\left.\left(\Phi, \xi^{*} \wedge \Phi^{\prime}\right)=(\xi\lrcorner \Phi, \Phi^{\prime}\right)
$$

(3.6) where $\xi\lrcorner \Phi \in \Lambda^{q-1}\left(\left(\mathbb{R}^{d}\right)^{*}\right)$ is determined by $\left.\left(\xi_{1}\right\lrcorner \Phi\right)\left(\xi_{2}, \ldots, \xi_{q}\right)=\Phi\left(\xi_{1}, \ldots, \xi_{q}\right) \quad$ for $\left(\xi_{1}, \ldots, \xi_{q}\right) \in\left(\mathbb{R}^{d}\right)^{q}$, 
we arrive at

$$
\left.\tilde{\delta} \Phi=-\sum_{i=1}^{d} \mathbf{e}_{i}\right\lrcorner\left(\mathfrak{E}\left(\mathbf{e}_{i}\right) \Phi\right) .
$$

In particular,

$$
\left.\widetilde{\delta \omega}=-\sum_{i=1}^{d} \mathbf{e}_{i}\right\lrcorner\left(\mathfrak{E}\left(\mathbf{e}_{i}\right) \tilde{\omega}\right) ;
$$

and so, if $\left(\left(E_{1}\right)_{x}, \ldots,\left(E_{d}\right)_{x}\right)$ is an orthonormal basis in $T_{x} M$, then

$$
\left.(\delta \omega)_{x}=-\sum_{i=1}^{d}\left(E_{i}\right)_{x}\right\lrcorner \nabla_{\left(E_{i}\right)_{x}} \omega
$$

where the meaning assigned to " $\lrcorner$ " here should be obvious by analogy with (3.6).

We are now ready to give an expression for the Hodge operator $H \equiv$ $\delta d+d \delta$. Indeed, by combining (3.4) with (3.8), we see that

$$
\left.-\widehat{\delta d \omega}=\sum_{i, j=1}^{d} \mathbf{e}_{j}\right\lrcorner\left(\mathbf{e}_{i}^{*} \wedge \mathfrak{E}\left(\mathbf{e}_{j}\right) \mathfrak{E}\left(\mathbf{e}_{i}\right) \tilde{\omega}\right)
$$

and

$$
\left.-\widetilde{d \delta \omega}=\sum_{i, j=1}^{d} \mathbf{e}_{i}^{*} \wedge\left(\mathbf{e}_{j}\right\lrcorner \mathfrak{E}\left(\mathbf{e}_{i}\right) \mathfrak{E}\left(\mathbf{e}_{j}\right) \tilde{\omega}\right),
$$

where we adopt the convention that $\xi\lrcorner \Phi=0$ when $\Phi \in \Lambda^{0}\left(\left(\mathbb{R}^{d}\right)^{*}\right)$. Next, for $1 \leq q \leq \operatorname{dim}(M), \Phi \in \Lambda^{q}\left(\left(\mathbb{R}^{d}\right)^{*}\right)$, and $\left(\xi_{1}, \ldots, \xi_{q}\right) \in \mathbb{R}^{d}$, observe that

$\left.\left[\mathbf{e}_{j}\right\lrcorner\left(\mathbf{e}_{i}^{*} \wedge \Phi\right)\right]\left(\xi_{1}, \ldots, \xi_{q}\right)=\left(\mathbf{e}_{i}, \mathbf{e}_{j}\right) \Phi\left(\xi_{1}, \ldots, \xi_{q}\right)$

$$
-\sum_{\mu=1}^{q}(-1)^{\mu-1}\left(\mathbf{e}_{i}, \xi_{\mu}\right) \Phi\left(\mathbf{e}_{j}, \xi_{1}, \ldots, \widehat{\xi_{\mu}}, \ldots, \xi_{q}\right)
$$

and

$$
\begin{aligned}
& \left.\left[\mathbf{e}_{i}^{*} \wedge\left(\mathbf{e}_{j}\right\lrcorner \Phi\right)\right]\left(\xi_{1}, \ldots, \xi_{q}\right) \\
& \quad=\sum_{\mu=1}^{q}(-1)^{\mu-1}\left(\mathbf{e}_{i}, \xi_{\mu}\right) \Phi\left(\mathbf{e}_{j}, \xi_{1}, \ldots, \widehat{\xi_{\mu}}, \ldots, \xi_{q}\right)
\end{aligned}
$$


Hence, after adding the preceding expressions for $-\widetilde{\delta d \omega}$ and $-\widetilde{d \delta \omega}$, we see that, for $\omega \in \Omega^{q}(M)$,

$$
\begin{aligned}
-[\widehat{H \omega}]\left(\xi_{1}, \ldots, \xi_{q}\right)= & \sum_{i=1}^{d}\left[\mathfrak{E}\left(\mathbf{e}_{i}\right)^{2} \tilde{\omega}\right]\left(\xi_{1}, \ldots, \xi_{q}\right) \\
& +\sum_{\mu=1}^{q} \sum_{j=1}^{d}(-1)^{\mu-1}\left[\left[\mathfrak{E}\left(\xi_{\mu}\right), \mathfrak{E}\left(\mathbf{e}_{j}\right)\right] \tilde{\omega}\right] \\
& \cdot\left(\mathbf{e}_{j}, \xi_{1}, \ldots, \widehat{\xi_{\mu}}, \ldots, \xi_{q}\right) .
\end{aligned}
$$

In order to take the next step, recall (cf. (8.44) in [13]) that

$$
\left[\mathfrak{E}\left(\mathbf{e}_{i}\right), \mathfrak{E}\left(\mathbf{e}_{j}\right)\right]=-\lambda\left(\Omega\left(\mathbf{e}_{i}, \mathbf{e}_{j}\right)\right),
$$

where $\mathfrak{f} \rightsquigarrow \Omega_{\mathfrak{f}}$ is the $o\left(\mathbb{R}^{d}\right)$-valued 2-form which is called the curvature 2-form because (cf. (8.54) in [13] and use $R(X, Y) Z \equiv\left[\nabla_{X}, \nabla_{Y}\right] Z-$ $\nabla_{[X, Y]} Z$ for the Riemann curvature)

$$
\Omega_{\mathfrak{f}}\left(\xi_{1}, \xi_{2}\right) \xi_{3}=\mathfrak{f}^{-1} R\left(\mathfrak{f} \xi_{1}, \mathfrak{f} \xi_{2}\right) \mathfrak{f} \xi_{3},
$$

and, for $a \in o\left(\mathbb{R}^{d}\right), \lambda(a)$ is the vertical vector field on $\mathcal{O}(M)$ given by (cf. (8.3) in [13])

$$
\lambda(a)_{\mathfrak{f}}=\left.\frac{d}{d t} R_{e^{t a}} \mathfrak{f}\right|_{t=0} .
$$

Thus, because, for $\eta \in \Omega^{q}(M)$,

$$
[\lambda(a) \tilde{\eta}]\left(\xi_{1}, \ldots, \xi_{q}\right)=\sum_{\nu=1}^{q} \tilde{\eta}\left(\xi_{1}, \ldots, a \xi_{\nu}, \ldots, \xi_{q}\right)
$$


we have that

$$
\begin{aligned}
& \sum_{\mu=1}^{q}(-1)^{\mu-1}\left[\left[\mathfrak{E}\left(\xi_{\mu}\right), \mathfrak{E}\left(\mathbf{e}_{j}\right)\right] \tilde{\omega}\right]\left(\mathbf{e}_{j}, \xi_{1}, \ldots, \widehat{\xi_{\mu}}, \ldots, \xi_{q}\right) \\
&=\sum_{\mu=1}^{q}(-1)^{\mu} \tilde{\omega}\left(\Omega\left(\xi_{\mu}, \mathbf{e}_{j}\right) \mathbf{e}_{j}, \xi_{1}, \ldots, \widehat{\xi_{\mu}}, \ldots, \xi_{q}\right) \\
& \quad+\sum_{1 \leq \nu<\mu \leq q}(-1)^{\mu+\nu} \tilde{\omega}\left(\mathbf{e}_{j}, \Omega\left(\xi_{\mu}, \mathbf{e}_{j}\right) \xi_{\nu}, \xi_{1}, \ldots \widehat{\xi_{\nu}}, \ldots, \widehat{\xi_{\mu}}, \ldots \xi_{q}\right) \\
& \quad-\sum_{1 \leq \mu<\nu \leq q}(-1)^{\mu+\nu} \tilde{\omega}\left(\mathbf{e}_{j}, \Omega\left(\xi_{\mu}, \mathbf{e}_{j}\right) \xi_{\nu}, \xi_{1}, \ldots \widehat{\xi_{\mu}}, \ldots, \widehat{\xi_{\nu}}, \ldots, \xi_{q}\right) \\
&=\sum_{\mu=1}^{q}(-1)^{\mu} \tilde{\omega}\left(\Omega\left(\xi_{\mu}, \mathbf{e}_{j}\right) \mathbf{e}_{j}, \xi_{1}, \ldots, \widehat{\xi_{\mu}}, \ldots, \xi_{q}\right) \\
& \quad+\sum_{1 \leq \nu<\mu \leq q}(-1)^{\mu+\nu} \tilde{\omega}\left(\mathbf{e}_{j}, \Omega\left(\xi_{\nu}, \xi_{\mu}\right) \mathbf{e}_{j}, \xi_{1}, \ldots, \widehat{\xi_{\mu}}, \ldots, \widehat{\xi_{\nu}}, \ldots \xi_{q}\right)
\end{aligned}
$$

where, in the passage to the last equation we have used the first Bianci identity to obtain

$$
\Omega\left(\xi_{\mu}, \mathbf{e}_{j}\right) \xi_{\nu}-\Omega\left(\xi_{\nu}, \mathbf{e}_{j}\right) \xi_{\mu}=\Omega\left(\xi_{\mu}, \xi_{\nu}\right) \mathbf{e}_{j}
$$

When we combine this with (3.11), we find that

$$
\begin{aligned}
- & {[\widetilde{H \omega}]_{\mathfrak{f}}\left(\xi_{1}, \ldots, \xi_{q}\right) } \\
& =\sum_{i=1}^{d}\left[\mathfrak{E}\left(\mathbf{e}_{i}\right)_{\mathfrak{f}}^{2} \tilde{\omega}\right]\left(\xi_{1}, \ldots, \xi_{q}\right) \\
& -\sum_{\mu=1}^{q}(-1)^{\mu-1} \sum_{i=1}^{d} \tilde{\omega}_{\mathfrak{f}}\left(\Omega_{\mathfrak{f}}\left(\xi_{\mu}, \mathbf{e}_{i}\right) \mathbf{e}_{i}, \xi_{1}, \ldots, \widehat{\xi_{\mu}}, \ldots, \xi_{q}\right) \\
& +\sum_{1 \leq \mu<\nu \leq q}(-1)^{\mu+\nu-1} \sum_{i=1}^{d} \tilde{\omega}_{\mathfrak{f}}\left(\Omega\left(\xi_{\mu}, \xi_{\nu}\right) \mathbf{e}_{i}, \mathbf{e}_{i} \xi_{1}, \ldots, \widehat{\xi_{\mu}}, \ldots, \widehat{\xi_{\nu}}, \ldots, \xi_{q}\right) .
\end{aligned}
$$

Now take Bochner's Laplacian (cf. (8.29) in [13])

$$
\Delta_{B} \equiv \sum_{i=1}^{d} \mathfrak{E}\left(\mathbf{e}_{i}\right)^{2}
$$


and recall (cf. (8.58) in [13]) that

$$
\Re_{\mathfrak{f}} \xi \equiv \sum_{i=1}^{d} \Omega_{\mathfrak{f}}\left(\xi, \mathbf{e}_{i}\right) \mathbf{e}_{i}=\mathfrak{f}^{-1} \operatorname{Ric}_{\pi \mathfrak{f}} \mathfrak{f} \xi
$$

where $x \in M \longmapsto \operatorname{Ric}_{x} \in \operatorname{Hom}\left(T_{x} M ; T_{x} M\right)$ is the Ricci curvature tensor. Further, for each $\mathfrak{f} \in \mathcal{O}(M)$, set

$$
\mathfrak{R}_{\mathfrak{f}}^{(*)}=\bigoplus_{q=0}^{\operatorname{dim}(M)} \mathfrak{R}_{\mathfrak{f}}^{(q)} \quad \text { and } \quad \Omega_{\mathfrak{f}}^{(*)}=\bigoplus_{q=0}^{\operatorname{dim}(M)} \Omega_{\mathfrak{f}}^{(q)}
$$

where, for each $0 \leq q \leq \operatorname{dim}(M), \mathfrak{R}_{\mathfrak{f}}^{(q)}$ and $\Omega_{\mathfrak{f}}^{(q)}$ are the linear maps on $\Lambda^{q}\left(\left(\mathbb{R}^{d}\right)^{*}\right)$ such that: $\mathfrak{R}_{\mathfrak{f}}^{(0)}=0$ and, for $1 \leq q \leq \operatorname{dim}(M)$ and $\Phi \in \Lambda^{q}\left(\left(\mathbb{R}^{d}\right)^{*}\right)$,

$$
\left[\mathfrak{R}_{\mathfrak{f}}^{(q)} \Phi\right]\left(\xi_{1}, \ldots, \xi_{q}\right)=\sum_{\mu=1}^{q}(-1)^{\mu-1} \Phi\left(\Re_{f} \xi_{\mu}, \xi_{1}, \ldots, \widehat{\xi_{\mu}}, \ldots, \xi_{q}\right)
$$

while $\Omega_{\mathfrak{f}}^{(0)}=0, \Omega_{\mathfrak{f}}^{(1)}=0$, and, for $2 \leq q \leq \operatorname{dim}(M)$ and $\Phi \in \Lambda^{q}\left(\left(\mathbb{R}^{d}\right)^{*}\right)$,

$$
\begin{aligned}
& {\left[\Omega_{\mathfrak{f}}^{(q)} \Phi\right]\left(\xi_{1}, \ldots, \xi_{q}\right)} \\
& \quad=\sum_{1 \leq \mu<\nu \leq q}(-1)^{\mu+\nu-1} \sum_{i=1}^{d} \Phi\left(\Omega_{\mathfrak{f}}\left(\xi_{\mu}, \xi_{\nu}\right) \mathbf{e}_{i}, \mathbf{e}_{i}, \xi_{1}, \ldots, \widehat{\xi_{\mu}}, \ldots, \widehat{\xi_{\nu}}, \ldots, \xi_{q}\right) .
\end{aligned}
$$

Then, if we define $\tilde{H}$ on $C^{2}\left(\mathcal{O}(M) ; \Lambda^{*}\left(\left(\mathbb{R}^{d}\right)^{*}\right)\right)$ so that

$$
-\tilde{H} \Phi=\Delta_{B} \Phi-\left(\mathfrak{R}^{(*)}-\Omega^{(*)}\right) \Phi,
$$

the preceding calculation of $\widetilde{H \omega}$ can be summarized by

$$
\widetilde{H \omega}=\tilde{H} \tilde{\omega} .
$$

For future reference, we want to record here two important properties of the operators $\Re^{(*)}$ and $\Omega^{(*)}$. In the first place, they are equivariant under the action of the rotation group. Namely, for $O \in \mathcal{O}\left(\mathbb{R}^{d}\right)$, define the linear transformation $R_{O}$ on $\Lambda^{*}\left(\left(\mathbb{R}^{d}\right)^{*}\right)$ so that

$$
\left[R_{O} \Phi\right]\left(\xi_{1}, \ldots, \xi_{q}\right)=\Phi\left(O \xi_{1}, \ldots, O \xi_{q}\right) \quad \text { if } \Phi \in \Lambda^{q}\left(\left(\mathbb{R}^{d}\right)^{*}\right) .
$$


Then

$$
\mathfrak{R}_{R_{O} f}^{(*)}=R_{O} \circ \Re_{\mathfrak{f}}^{(*)} \circ R_{O}^{-1} \quad \text { and } \quad \Omega_{R_{O f}}^{(*)}=R_{O} \circ \Omega_{f}^{(*)} \circ R_{O}^{-1} .
$$

To see these, note, for instance, that if $1 \leq q \leq \operatorname{dim}(M)$ and $\Phi \in$ $\Lambda^{q}\left(\left(\mathbb{R}^{d}\right)^{*}\right.$ ), then, because (cf. (8.59) in [13]) $\mathfrak{R}_{R_{O} \mathfrak{f}}=O^{\top} \mathfrak{R}_{\mathfrak{f}} O$,

$$
\begin{aligned}
{\left[R_{O} \circ \Re^{(q)} \Phi\right]\left(\xi_{1}, \ldots, \xi_{q}\right) } & =\sum_{\mu=1}^{q}(-1)^{\mu-1} R_{O} \Phi\left(O^{\top} \mathfrak{R}_{\mathfrak{f}} O \xi_{\mu}, \xi_{1}, \ldots, \widehat{\xi_{\mu}}, \ldots, \xi_{q}\right) \\
& =\left[\mathfrak{R}_{\mathfrak{f}}^{(q)} \circ R_{O} \Phi\right]\left(\xi_{1}, \ldots, \xi_{q}\right) .
\end{aligned}
$$

The corresponding result for $\Omega^{(q)}$ with $2 \leq q \leq \operatorname{dim}(M)$ follows in a similar way from (cf. (8.50) in [13]) $\Omega_{R_{O}}\left(\xi, \xi^{\prime}\right)=O^{\top} \Omega_{\mathfrak{f}}\left(O \xi, O \xi^{\prime}\right) O$. Secondly, they are both symmetric in the sense that

$$
\left(\Phi^{\prime}, \mathfrak{R}^{(*)} \Phi\right)=\left(\Phi, \mathfrak{R}^{(*)} \Phi^{\prime}\right) \quad \text { and } \quad\left(\Phi^{\prime}, \Omega^{(*)} \Phi\right)=\left(\Phi, \Omega^{(*)} \Phi^{\prime}\right) .
$$

In case it is not clear how the operators $\mathfrak{R}^{(q)}$ and $\Omega^{(q)}$ are related to more familiar manifestations of the Riemann curvature, first note that

$$
\left[\Re_{\mathfrak{f}}^{(1)} \tilde{\omega}_{\mathfrak{f}}\right](\xi)=\omega_{\pi \mathfrak{f}}\left(\operatorname{Ric}_{\pi \mathfrak{f}} \mathfrak{f} \xi\right) \quad \text { for } \mathfrak{f} \in \mathcal{O}(M) \text { and } \xi \in \mathbb{R}^{\operatorname{dim}(M)}
$$

$$
\begin{aligned}
{\left[\Re^{(q)} \tilde{\omega}\right]\left(\xi_{1}, \ldots, \xi_{q}\right) } & =\sum_{\mu=1}^{q}\left[\Re^{(1)} \omega^{(\mu)}\left(\xi_{1}, \ldots, \widehat{\xi_{\mu}}, \ldots, \xi_{q}\right)\right]\left(\xi_{\mu}\right) \\
\text { for } 2 & \leq q \leq \operatorname{dim}(M) \text { and } \xi_{1}, \ldots, \xi_{q} \in\left(\mathbb{R}^{\operatorname{dim}(M)}\right)^{q},
\end{aligned}
$$

where $\tilde{\omega}_{\mathfrak{f}}^{(\mu)} \in \operatorname{Hom}\left(\Lambda^{q-1} \mathbb{R}^{\operatorname{dim} M} ; \Lambda^{1} \mathbb{R}^{\operatorname{dim}(M)}\right)$ is determined so that

$$
\left[\tilde{\omega}_{\mathfrak{f}}^{(\mu)}\left(\xi_{1}, \ldots, \widehat{\xi_{\mu}}, \ldots, \xi_{q}\right)\right]\left(\xi_{\mu}\right)=\tilde{\omega}\left(\xi_{1}, \ldots, \xi_{q}\right) \text {. }
$$

Similarly, recall that the Riemann curvature operator

$$
R_{x}^{\mathrm{op}} \in \operatorname{Hom}\left(\Lambda^{2}\left(T_{x} M^{*}\right) ; \Lambda^{2}\left(T_{x} M^{*}\right)\right)
$$

is defined so that, for any orthonormal basis $\left(\left(E_{1}\right)_{x}, \ldots,\left(E_{d}\right)_{x}\right)$ in $T_{x} M$,

$$
\left[R_{x}^{\mathrm{op}}\left(\omega_{x}\right)\right]\left(X_{x}, Y_{x}\right)=\sum_{i, j=1}^{d}\left(R\left(\left(E_{i}\right)_{x},\left(E_{j}\right)_{x}\right) Y_{x}, X_{x}\right) \omega_{x}\left(\left(E_{i}\right)_{x},\left(E_{j}\right)_{x}\right)
$$


for $X_{x}, Y_{x} \in T_{x} M$. Then,

$$
\begin{aligned}
& \Omega_{\mathfrak{f}}^{(2)} \tilde{\omega}_{\mathfrak{f}}=R_{\pi \mathfrak{f}}^{o p} \omega_{\pi \mathfrak{f}}, \quad \text { and when } 3 \leq q \leq \operatorname{dim}(M): \\
& {\left[\begin{array}{l}
\left.\Omega^{(q)} \tilde{\omega}\right]\left(\xi_{1}, \ldots, \xi_{q}\right) \\
\quad=\sum_{1 \leq \mu<\nu \leq q}\left[\Omega^{(2)} \tilde{\omega}^{(\mu, \nu)}\left(\xi_{1}, \ldots, \widehat{\xi_{\mu}}, \ldots, \widehat{\xi_{\nu}}, \ldots, \xi_{q}\right)\right]\left(\xi_{\mu}, \xi_{\nu}\right),
\end{array}\right.}
\end{aligned}
$$

where $\tilde{\omega}_{\mathfrak{f}}^{(\mu, \nu)} \in \operatorname{Hom}\left(\Lambda^{q-2} \mathbb{R}^{\operatorname{dim} M} ; \Lambda^{2} \mathbb{R}^{\operatorname{dim}(M)}\right)$ is determined so that

$$
\left[\tilde{\omega}_{f}^{(\mu, \nu)}\left(\xi_{1}, \ldots, \widehat{\xi_{\mu}}, \ldots, \widehat{\xi_{\nu}}, \ldots, \xi_{q}\right)\right]\left(\xi_{\mu}, \xi_{\nu}\right)=\tilde{\omega}\left(\xi_{1}, \ldots, \xi_{q}\right) .
$$

Before closing this section, we want to see how the Hodge operator is altered when we compute the formal adjoint $\delta^{U}$ of $d$ with respect to (cf. (1.3)) $m^{U}$ instead of $\lambda_{M}$. But clearly

$$
\left.\delta^{U} \omega=e^{U} \delta\left(e^{-U} \omega\right)=\delta \omega+\operatorname{grad} U\right\lrcorner \omega .
$$

We next want to lift these considerations to $\mathcal{O}(M)$. For this purpose, set $\tilde{U}=U \circ \pi$ and define $\widetilde{m^{U}}$ on $\mathcal{O}(M)$ so that $\widetilde{d m^{U}}=e^{-\tilde{U}} d \lambda_{\mathcal{O}(M)}$. Then the analogous calculation shows that the formal adjoint $\widetilde{\delta^{U}}$ of $\tilde{d}$ with respect to $\widetilde{m^{U}}$ is given by

$$
\left.\widetilde{\delta^{U}} \Phi=\tilde{\delta} \Phi+\operatorname{Grad} \tilde{U}\right\lrcorner \Phi,
$$

where, for any $F \in C^{1}(\mathcal{O}(M) ; \mathbb{R})$,

$$
\operatorname{Grad} F \equiv \sum_{i=1}^{d}\left(\mathfrak{E}\left(\mathbf{e}_{i}\right) F\right) \mathbf{e}_{i}
$$

and clearly $\widetilde{\delta^{U} \omega}=\widetilde{\delta^{U}} \tilde{\omega}$. In particular,

$$
\left.\left.\widetilde{\left(\delta^{U}-\delta\right.}\right) d \omega=\sum_{i, j=1}^{d}\left(\mathfrak{E}\left(\mathbf{e}_{j}\right) \tilde{U}\right) \mathbf{e}_{j}\right\lrcorner\left(\mathbf{e}_{i}^{*} \wedge \mathfrak{E}\left(\mathbf{e}_{i}\right) \tilde{\omega}\right)
$$

and

$$
\begin{aligned}
d\left(\widehat{\delta^{U}-\delta}\right) \omega= & \left.\sum_{i, j=1}^{d} \mathbf{e}_{i}^{*} \wedge \mathfrak{E}\left(\mathbf{e}_{i}\right)\left(\mathfrak{E}\left(\mathbf{e}_{j}\right) \tilde{U} \mathbf{e}_{j}\right\lrcorner \tilde{\omega}\right) \\
= & \left.\sum_{i, j} \mathfrak{E}\left(\mathbf{e}_{i}\right) \mathfrak{E}\left(\mathbf{e}_{j}\right) \tilde{U} \mathbf{e}_{i}^{*} \wedge\left(\mathbf{e}_{j}\right\lrcorner \tilde{\omega}\right) \\
& \left.+\sum_{i, j=1}^{d}\left(\mathfrak{E}\left(\mathbf{e}_{j}\right) \tilde{U}\right) \mathbf{e}_{i}^{*} \wedge\left(\mathbf{e}_{j}\right\lrcorner \mathfrak{E}\left(\mathbf{e}_{i}\right) \tilde{\omega}\right)
\end{aligned}
$$


Thus, if we define $\mathfrak{f} \rightsquigarrow \operatorname{Hess}_{\mathfrak{f}} \tilde{U} \in \operatorname{Hom}\left(\mathbb{R}^{d} ; \mathbb{R}^{d}\right)$ so that

$$
\operatorname{Hess}_{\mathfrak{f}} \tilde{U} \xi=\sum_{i=1}^{d}\left(\mathfrak{E}(\xi)_{\mathfrak{f}} \mathfrak{E}\left(\mathbf{e}_{i}\right) \tilde{U}\right) \mathbf{e}_{i},
$$

and if we define $\left(\mathfrak{R}^{U}\right)_{\mathfrak{f}}^{(*)}$ on $\Lambda^{*}\left(\left(\mathbb{R}^{d}\right)^{*}\right)$ in terms of

$$
\Re_{\mathfrak{f}}^{U} \equiv \mathfrak{R}_{\mathfrak{f}}+\operatorname{Hess}_{\mathfrak{f}} \tilde{U}
$$

in the same way (cf. (3.19)) as we previously defined $\mathfrak{R}_{\mathfrak{f}}^{(*)}$ from $\mathfrak{R}_{\mathfrak{f}}$, then, by the considerations made earlier (especially (3.9) and (3.10)), we see that the Hodge Laplacian with weight $U$

$$
H^{U} \equiv \delta^{U} d+d \delta^{U}
$$

satisfies

$$
\begin{aligned}
& \widehat{H^{U} \omega}=\widehat{H^{U}} \tilde{\omega} \\
& \text { where }-\widehat{H^{U}} \Phi=\Delta_{B} \Phi-\mathfrak{E}(\operatorname{Grad} \tilde{U}) \Phi-\left(\left(\Re^{U}\right)^{(*)}-\Omega^{(*)}\right) \Phi .
\end{aligned}
$$

Notice that, because $\operatorname{Hess}_{R_{O} f} \tilde{U}=O^{\top}\left(\operatorname{Hess}_{\mathfrak{f}} \tilde{U}\right) O$ and $\operatorname{Hess}_{\mathfrak{f}} \tilde{U}$ is symmetric, the first equations in (3.17) and (3.18) admit the following extensions:

$$
\begin{gathered}
\left(\Re^{U}\right)_{R_{O} f}^{(*)}=R_{O} \circ\left(\Re^{U}\right)_{\mathfrak{f}}^{(*)} \circ R_{O}^{-1} \\
\text { and } \quad\left(\Phi^{\prime},\left(\Re^{U}\right)_{\mathfrak{f}}^{(*)} \Phi\right)=\left(\Phi,\left(\Re^{U}\right)_{\mathfrak{f}}^{(*)} \Phi^{\prime}\right)
\end{gathered}
$$

\section{Heat flow for the Hodge operator}

In order to combine the considerations in $\S 3$ with those in the sections preceding it, we must first lift the diffusion for the operator $L^{U}$ to $\mathcal{O}(M)$. That is, let $U: M \longrightarrow[0, \infty)$ be a smooth function which satisfies the condition in $(1.1)$, and define the operator $\widetilde{L^{U}}$ on $C^{2}(\mathcal{O}(M) ; \mathbb{R})$ so that (cf. (3.13))

$$
\widetilde{L^{U}} F=e^{\tilde{U}} \sum_{i=1}^{d} \mathfrak{E}\left(\mathbf{e}_{i}\right)\left(e^{-\tilde{U}} \mathfrak{E}\left(\mathbf{e}_{i}\right) F\right)=\Delta_{B} F-\mathfrak{E}(\operatorname{Grad} \tilde{U}) F
$$


By starting with $U$ 's which have compact support and repeating ${ }^{9}$ the procedure used in $\S 1$, one can show that, for a given $\mathfrak{f} \in \mathcal{O}(M)$, there exists precisely one Borel probability measure $\widetilde{\mathbb{P}_{\mathfrak{f}}^{U}}$ on

$$
\mathcal{P}(\mathcal{O}(M)) \equiv C([0, \infty) ; \mathcal{O}(M))
$$

with the properties that $\widetilde{\mathbb{P}_{\mathfrak{f}}^{U}}(\mathfrak{p}(0)=\mathfrak{f})=1$ and, for any $F \in C_{\mathrm{c}}^{\infty}(\mathcal{O}(M) ; \mathbb{R})$,

$$
F(\mathfrak{p}(t))-\int_{0}^{t} \widetilde{L^{U}} F(\mathfrak{p}(\tau)) d \tau \quad \text { is a } \widetilde{\mathbb{P}_{\mathfrak{f}}^{U}} \text {-martingale }
$$

relative to $\left\{\widetilde{\mathcal{B}}_{t}: t>0\right\}$, where $\widetilde{\mathcal{B}}_{t}=\sigma(\{\mathfrak{p}(\tau): \tau \in[0, t]\})$. In fact (cf. (1.11)), for each $T \in[0, \infty)$ and all $\widehat{\mathcal{B}}_{T}$-measurable $F: \mathcal{P}(\mathcal{O}(M)) \longrightarrow$ $[0, \infty)$

$$
\mathbb{E}^{\widetilde{\mathbb{P}_{f}^{U}}}[F(\mathfrak{p})]=\mathbb{E}^{\widetilde{\mathbb{P}^{0}}}\left[\widetilde{R^{U}}(T, \mathfrak{p}) F(\mathfrak{p})\right]
$$

where $\widetilde{R^{U}}(T, \mathfrak{p}) \equiv R^{U}(T, \pi \circ \mathfrak{p})$. In particular, either directly from (4.2) or by using (cf. (8.28) in [13])

$$
\widetilde{L^{U}}(\varphi \circ \pi)=\left(L^{U} \varphi\right) \circ \pi, \quad \varphi \in C^{2}(M ; \mathbb{R}),
$$

one sees that the distribution $\pi_{*} \widetilde{\mathbb{P}_{\mathfrak{f}}^{U}}$ of $\mathfrak{p} \rightsquigarrow \pi$ o $\mathfrak{p}$ under $\widetilde{\mathbb{P}_{\mathfrak{f}}^{U}}$ must be $\mathbb{P}_{\pi \mathfrak{f}}^{U}$. Similarly, either from uniqueness or from (4.2) combined with (8.38) in [13], one sees that

$$
\widetilde{\mathbb{P}_{R_{O}}^{U}}=\left(R_{O}\right)_{*} \widetilde{\mathbb{P}_{\mathfrak{f}}^{U}}
$$

Furthermore, the mapping $\mathfrak{f} \in \mathcal{O}(M) \longmapsto \widetilde{\mathbb{P}_{f}^{U}} \in \mathbf{M}_{1}(\mathcal{P}(\mathcal{O}(M)))$ is weakly continuous, and the family $\left\{\widetilde{\mathbb{P}_{f}^{U}}: \mathfrak{f} \in \mathcal{O}(M)\right\}$ satisfies the obvious analog of the Markov property explained in (2.1). Finally, if

$$
\widetilde{\mathbb{P}^{U}} \equiv \int_{\mathcal{O}(M)} \widetilde{\mathbb{P}_{f}^{U}} \widetilde{m^{U}}(d f)
$$

then, starting from (4.2), using the fact (cf. 8.35 in [13]) that $\mathbb{P}^{0}$ is reversible, and proceeding in exactly the same way as in the derivation

\footnotetext{
${ }^{9}$ Observe that the argument is simpler here because there are no annoyances arising from lack of smoothness.
} 
of (1.8), we see that $\widetilde{\mathbb{P}^{U}}$ is reversible, in that the sense that the obvious analog of (1.8) holds.

To take the next step, we must add further assumptions. In the first place, we need to assume that there exists a $\kappa_{\mathrm{op}} \in[0, \infty)$ such that (cf. $(3.20))$

$$
\left\langle R_{x}^{\mathrm{op}} \omega_{x}, \omega_{x}\right\rangle \leq \kappa_{\mathrm{op}}\left\|\omega_{x}\right\|^{2} \quad \text { for all } x \in M \text { and } \omega_{x} \in \Lambda^{2}\left(\left(\mathbb{R}^{d}\right)^{*}\right) .
$$

Secondly, and even more restrictive, we need to require that the sum of the Ricci tensor and the Hessian of $U$ is uniformly bounded below. That is, we must assume that there exists a $\kappa_{U} \in[0, \infty)$ such that (cf. $(3.21))$

$$
\left(\mathfrak{R}_{\mathfrak{f}}^{U} \xi, \xi\right) \geq-\kappa_{U}|\xi|^{2} \quad \text { for all } \mathfrak{f} \in \mathcal{O}(M) \text { and } \xi \in \mathbb{R}^{d} \text {. }
$$

The reason for our making these assumptions is that we want to know that there exists a map

$$
\left(\widetilde{m^{U}}\right)^{(*)}:[0, \infty) \times \mathcal{P}(\mathcal{O}(M)) \longrightarrow \operatorname{Hom}\left(\Lambda^{*}\left(\left(\mathbb{R}^{d}\right)^{*}\right) ; \Lambda^{*}\left(\left(\mathbb{R}^{d}\right)^{*}\right)\right)
$$

such that

$$
\begin{aligned}
& \left.\widetilde{\left(m^{U}\right.}\right)^{(*)}(t, \mathfrak{p})=I_{\Lambda^{*}\left(\left(\mathbb{R}^{d}\right)^{*}\right)} \\
& -\int_{0}^{t}\left(\widetilde{m^{U}}\right)^{(*)}(\tau, \mathfrak{p})\left(\left(\mathfrak{R}_{\mathfrak{p}(\tau)}^{U}\right)^{(*)}-\Omega_{\mathfrak{p}(\tau)}^{(*)}\right) d \tau .
\end{aligned}
$$

Because of (3.19), it should be clear that (4.4) and (4.5) are sufficient to guarantee not only that, for each $\mathfrak{p} \in \mathcal{P}(\mathcal{O}(M))$ ), there exists a unique solution to (4.6) but also that this solution is a continuous function of $(t, \mathfrak{p})$ with the properties that, for each $T \in[0, \infty), \mathfrak{p} \rightsquigarrow\left(\widetilde{m^{U}}\right)^{(*)}(T, \mathfrak{p})$ depends only on $\mathfrak{p}\lceil[0, T]$ and (cf. (3.19) and (3.20))

$$
\begin{array}{r}
\left\|\widetilde{\left.m^{U}\right)^{(*)}}(T, \mathfrak{p})\right\|_{\mathrm{op}} \leq e^{C_{U} T}, \quad(T, \mathfrak{p}) \in[0, \infty) \times \mathcal{P}(\mathcal{O}(M)), \\
\text { where } C_{U} \equiv \operatorname{dim}(M)\left(\kappa_{U}+\frac{1}{2}(\operatorname{dim}(M)-1) \kappa_{\mathrm{op}}\right) .
\end{array}
$$

(For sharper estimates on the operator $\Re^{(*)}-\Omega^{(*)}$, see $\S 3$ of [7].) In fact, given any $T \in(0, \infty)$ and any family $\left\{T_{k, n}: n \geq 1 \& 0 \leq k \leq n\right\}$ such that

$$
0=T_{0, n} \leq \cdots \leq T_{n, n}=T \quad \text { and } \quad \lim _{n \rightarrow \infty} \max _{1 \leq k \leq n}\left(T_{k, n}-T_{k-1, n}\right)=0,
$$




$$
\begin{aligned}
\left(\widetilde{m^{U}}\right)^{(*)}(T, \mathfrak{p})=\lim _{n \rightarrow \infty} & \exp \left(-\tau_{1, n}\left(\left(\Re^{U}\right)^{(*)}-\Omega^{(*)}\right)_{\mathfrak{p}\left(T_{1, n}\right)}\right) \\
& \cdots \cdots \exp \left(-\tau_{n, n}\left(\left(\mathfrak{R}^{U}\right)^{(*)}-\Omega^{(*)}\right)_{\mathfrak{p}\left(T_{n, n}\right)}\right),
\end{aligned}
$$

where $\tau_{k, n} \equiv T_{k, n}-T_{k-1, n}$. In particular, this leads to (cf. (2.1))

$$
\left(\widetilde{m^{U}}\right)^{(*)}\left(t_{1}+t_{2}, \mathfrak{p}\right)=\left(\widetilde{m^{U}}\right)^{(*)}\left(t_{1}, \mathfrak{p}\right) \circ\left(\widetilde{m^{U}}\right)^{(*)}\left(t_{2}, \Sigma_{t_{1}} \mathfrak{p}\right)
$$

and, in conjunction with (3.17), (3.18), and (3.24), to

$$
\begin{array}{r}
\left.\left(\widetilde{m^{U}}\right)^{(*)}\left(T, R_{O} \circ \mathfrak{p}\right)=R_{O} \circ \widetilde{\left(m^{U}\right.}\right)^{(*)}(T, \mathfrak{p}) \circ R_{O}^{-1}, \\
(T, \mathfrak{p}) \in[0, \infty) \times \mathcal{P}(\mathcal{O}(M)) \& O \in \mathcal{O}\left(\mathbb{R}^{d}\right)
\end{array}
$$

and (cf. the last part of Theorem 1.4)

$$
\left.\left.\widetilde{\left(m^{U}\right.}\right)^{(*)}\left(T, \check{\mathfrak{p}}^{T}\right)=\widetilde{\left(m^{U}\right.}\right)^{(*)}(T, \check{\mathfrak{p}})^{\top},(T, \mathfrak{p}) \in[0, \infty) \times \mathcal{P}(\mathcal{O}(M))
$$

Our interest in $\widetilde{\mathbb{P}_{f}^{U}}$ and $\widetilde{M^{U}}$ stems from the fact that, for any smooth $\Phi: \mathcal{O}(M) \longrightarrow \Lambda^{*}\left(\left(\mathbb{R}^{d}\right)^{*}\right)$ with compact support,

$$
\begin{aligned}
& \left(\widetilde{M^{U}}\right)^{(*)}(T, \mathfrak{p}) \Phi_{\mathfrak{p}(t)}+\int_{0}^{t}\left(\widetilde{M^{U}}\right)^{(*)}(\tau, \mathfrak{p})\left(\widetilde{H^{U}} \Phi\right)_{\mathfrak{p}(\tau)} d \tau \\
& \text { is a } \Lambda^{*}\left(\left(\mathbb{R}^{d}\right)^{*}\right) \text {-valued } \widetilde{\mathbb{P}_{f}^{U}} \text {-martingale. }
\end{aligned}
$$

The proof is a simple application of (3.22) and the product formula (e.g., Lemma 2.41 in [13]) applied to the product of the continuously differentiable process $t \rightsquigarrow\left(\widetilde{M^{U}}\right)^{(*)}(t, \mathfrak{p})$ with the $\widetilde{\mathbb{P}_{\mathfrak{f}}^{U}}$-martingale

$$
\left.t \rightsquigarrow \Phi_{\mathfrak{p}(t)}-\int_{0}^{t} \widehat{\left(L^{U}\right.} \Phi\right)_{\mathfrak{p}(\tau)} d \tau
$$

Thus, if we define $\widetilde{\mathbf{Q}_{t}^{U}}$ on $C_{\mathrm{b}}\left(\mathcal{O}(M) ; \Lambda^{*}\left(\left(\mathbb{R}^{d}\right)^{*}\right)\right)$ so that

$$
\left(\widetilde{\mathbf{Q}_{t}^{U}} \Phi\right)_{\mathfrak{f}}=\widetilde{\mathbb{E}_{f}^{U}}\left[\widetilde{M^{U}}(t, \mathfrak{p}) \Phi_{\mathfrak{p}(t)}\right]
$$

then, by the Markov property for $\left\{\widetilde{\mathbb{P}_{\mathfrak{f}}^{U}}: \mathfrak{f} \in \mathcal{O}(M)\right\}$ and (4.8), $\left\{\widetilde{\mathbf{Q}_{t}^{U}}: t>0\right\}$ is a semigroup, and, by (4.11),

$$
\widetilde{\mathbf{Q}_{t}^{U}} \Phi=\Phi-\int_{0}^{t} \widetilde{\mathbf{Q}_{\tau}^{U}} \odot \widetilde{H^{U}} \Phi d \tau
$$


In addition, it follows from the reversibility of $\widetilde{\mathbb{P}^{U}}$ and (4.10) that

$$
\left(\Phi^{\prime}, \widetilde{\mathbf{Q}_{t}^{U}} \Phi\right)_{L^{2}\left(\widetilde{m^{U}}\right)}=\left(\Phi, \widetilde{\mathbf{Q}_{t}^{U}} \Phi^{\prime}\right)_{L^{2}\left(\widetilde{m^{U}}\right)}
$$

and (4.3) combined with (4.9) leads to

$$
\left(\widetilde{\mathbf{Q}_{t}^{U}} \Phi\right)_{R_{O} \mathfrak{f}}=R_{O}\left(\widetilde{\mathbf{Q}_{t}^{U}} \circ R_{O}^{-1} \Phi_{R_{O}}\right)_{\mathfrak{f}}, \quad \omega \in C_{\mathrm{b}}\left(M ; \Lambda^{*}\left(T M^{*}\right)\right),
$$

where $\left(\Phi_{R_{O}}\right)_{\mathfrak{f}} \equiv \Phi_{R_{O} f}$. Finally, by (4.7), it is clear that

$$
\left|\left(\widetilde{\mathbf{Q}_{t}^{U}} \Phi\right)_{\mathfrak{f}}\right| \leq e^{C_{U} t} \widetilde{\mathbb{E}^{\mathbb{P}}}\left[\left|\Phi_{\mathfrak{p}(t)}\right|\right]
$$

In preparation for the next step, observe that if $\omega \in C_{\mathrm{b}}\left(M ; \Lambda^{*}\left(T M^{*}\right)\right)$ then, because $\tilde{\omega}_{R_{O}}=R_{O} \tilde{\omega},(4.15)$ says that

$$
\left(\widetilde{\mathbf{Q}_{t}^{U}} \widetilde{\omega}\right)_{R_{O} \mathfrak{f}}=\left(R_{O} \circ \widetilde{\mathbf{Q}_{t}^{U}} \tilde{\omega}\right)_{\mathfrak{f}}
$$

Hence, there is a well defined operator $\mathbf{Q}_{t}^{U}$ on $C_{\mathrm{b}}\left(M ; \Lambda^{*}\left(T M^{*}\right)\right)$ such that

$$
\left(\widetilde{\mathbf{Q}_{t}^{U} \omega}\right)_{\mathfrak{f}}=\left(\widetilde{\mathbf{Q}_{t}^{U}} \tilde{\omega}\right)_{\mathfrak{f}}
$$

and clearly the semigroup property for $\left\{\mathbf{Q}_{t}^{U}: t>0\right\}$ follows from that for $\left\{\widehat{\mathbf{Q}_{t}^{U}}: t>0\right\}$. In addition, it follows from (4.14) that

$$
\left\langle\omega^{\prime}, \mathbf{Q}_{t}^{U} \omega\right\rangle_{L^{2}\left(m^{U}\right)}=\left\langle\omega, \mathbf{Q}_{t}^{U} \omega^{\prime}\right\rangle_{L^{2}\left(m^{U}\right)}
$$

where

$$
\left\langle\omega^{\prime}, \omega\right\rangle_{L^{2}\left(m^{U}\right)} \equiv \int_{M}\left\langle\omega^{\prime}, \omega\right\rangle d m^{U} .
$$

Also, from (4.16), we see that

$$
\left\|\left(\mathbf{Q}_{t}^{U} \omega\right)_{x}\right\| \leq e^{C_{U} t}\left[\mathbf{P}_{t}^{U}\|\omega\|\right](x),
$$

Finally, by (3.22) and (4.13),

$$
\mathbf{Q}_{t}^{U} \omega=\omega-\int_{0}^{t} \mathbf{Q}_{\tau}^{U} \circ H^{U} \omega d \tau \quad \text { for } t>0 \text { and } \omega \in \Omega_{\mathrm{c}}^{*}(M)
$$

We close this section with the following application of elliptic regularity. 
Theorem 4.21. Let $x \in M \longmapsto \omega_{x} \in \Lambda^{*}\left(T_{x} M^{*}\right)$ be bounded and continuous. Then $(t, x) \in[0, \infty) \times M \longmapsto\left(\omega_{t}\right)_{x} \in \Lambda^{*}\left(T M^{*}\right)$ is a bounded, continuous map which is smooth on $(0, \infty) \times M$ and satisfies

$$
\partial_{t} \omega_{t}+H^{U} \omega_{t}=0 \quad \text { with } \omega_{0}=\omega
$$

if and only if $\omega_{t}=\mathbf{Q}_{t}^{U} \omega$.

Proof. We first show that if $(t, x) \rightsquigarrow\left(\omega_{t}\right)_{x}$ satisfies the stated properties, then $\omega_{t}=\mathbf{Q}_{t}^{U} \omega$. To this end let $T \in(0, \infty)$ be given, and set

$$
\Phi_{t}(\mathfrak{p})=\widetilde{M^{U}}(t, \mathfrak{p})\left(\widetilde{\mathbf{Q}_{t}^{U}} \tilde{\omega}_{T-t}\right)_{\mathfrak{p}(t)} \text { for } t \in[0, T] .
$$

Then, from the martingale property in (4.11) and Doob's Stopping Time Theorem, one can check that, for any $r>0$,

$$
t \in[0, T] \longmapsto \Phi_{t \wedge \zeta_{r}}(\mathfrak{p}) \in \Lambda^{*}\left(\left(\mathbb{R}^{d}\right)^{*}\right)
$$

is a $\widetilde{\mathbb{P}_{f}^{U}}$-martingale, where

$$
\zeta_{r}(\mathfrak{p})=\inf \{t \geq 0: \operatorname{dist}(\pi \circ \mathfrak{p}(t), \pi \circ \mathfrak{p}(0)) \geq r\}
$$

Hence,

$$
\left(\tilde{\omega}_{T}\right)_{\mathfrak{f}}=\widehat{\mathbb{E}^{\mathbb{P}_{\mathfrak{f}}^{U}}}\left[\widetilde{M^{U}}\left(T \wedge \zeta_{r}(\mathfrak{p}), \mathfrak{p}\right)\left(\tilde{\omega}_{T-\zeta_{r}(\mathfrak{p})}\right)_{\mathfrak{p}\left(T \wedge \zeta_{r}\right)}\right] .
$$

Thus, after letting $r \rightarrow \infty$, we conclude that $\left(\tilde{\omega}_{T}\right)_{\mathfrak{f}}=\left(\widetilde{\mathbf{Q}_{T}^{U}} \tilde{\omega}\right)_{\mathfrak{f}}$, which is equivalent to $\omega_{T}=\mathbf{Q}_{T}^{U} \omega$.

Next, set $\omega_{t}=\mathbf{Q}_{t}^{U} \omega$. Clearly $\omega_{t} \longrightarrow \omega$ uniformly on compacts as $t \searrow 0$. Thus, by elliptic regularity theory, we will know that $(t, x) \times M \in$ $(0, \infty) \longmapsto\left(\omega_{t}\right)_{x} \in \Lambda^{*}\left(T_{x} M^{*}\right)$ is a smooth solution to (4.22) as soon as we show that, for each $\omega^{\prime} \in \Omega_{\mathrm{c}}^{*}(M)$,

$$
\frac{d}{d t}\left\langle\omega^{\prime}, \omega_{t}\right\rangle_{L^{2}\left(m^{U}\right)}+\left\langle H^{U} \omega^{\prime}, \omega_{t}\right\rangle_{L^{2}\left(m^{U}\right)}=0
$$

But, clearly this follows once we combine (4.18) with (4.20). q.e.d.

\section{A Hodge decomposition theorem}

The primary goal of this section is to prove the following Hodge decomposition result. 
Theorem 5.1. Assume that (4.4) holds and that $U \in C^{\infty}(M ;[0, \infty))$ satisfies the conditions in Theorem 2.11 and (4.5). Set $\operatorname{ker}\left(H^{U}\right)=\{\omega \in$ $\left.\Omega^{*}(M): H^{U} \omega=0\right\}$. Then, $K_{U}^{*}(M) \equiv \operatorname{ker}\left(H^{U}\right) \cap L^{2}\left(m^{U} ; \Lambda^{*}\left(T M^{*}\right)\right)$ is finite dimensional, and each of its elements is bounded. Moreover, for each closed $\omega \in \Omega^{*}(M) \cap L^{2}\left(m^{U} ; \Lambda^{*}\left(T M^{*}\right)\right)$, the orthogonal projection of $\omega$ onto $K_{U}^{*}(M)$ is the unique $\eta \in K_{U}^{*}(M)$ such that $\omega-\eta=d \xi$ for some $\xi \in \Omega^{*}(M) \cap L^{2}\left(m^{U} ; \Lambda^{*}\left(T M^{*}\right)\right)$. In fact, $\|\eta\|_{L^{2}(m U)} \leq\|\xi\|_{L^{2}\left(m^{U}\right)}$ and there is precisely one $\xi \in \Omega^{*}(M) \cap L^{2}\left(m^{U} ; \Lambda^{*}\left(T M^{*}\right)\right)$ such that $\xi \perp K_{U}^{*}(M), \delta^{U} \xi=0$, and $\omega-d \xi \in K_{U}^{*}(M)$. Finally,

$$
\alpha \equiv \inf \left\{\left\langle\omega, \overline{H^{U}} \omega\right\rangle_{L^{2}\left(m^{U}\right)}: \omega \perp K_{U}^{*}(M) \&\|\omega\|_{L^{2}\left(m^{U}\right)}=1\right\}>0
$$

and the preceding $\xi$ satisfies

$$
\|\xi\|_{L^{2}\left(m^{U}\right)} \leq \alpha^{-\frac{1}{2}}\|\omega-\eta\|_{L^{2}\left(m^{U}\right)} \leq \alpha^{-\frac{1}{2}}\|\omega\|_{L^{2}\left(m^{U}\right)} .
$$

Given the information which we have from $\S 1-\S 4$, the proof of Theorem 5.1 is mostly a matter of bookkeeping. Indeed, the crucial fact which we will need is contained in the following application of (2.12) and (4.19).

Lemma 5.2. For each $T \in(0, \infty)$

$$
\begin{array}{r}
\left\|\mathbf{Q}_{T}^{U} \omega\right\|_{L^{2}\left(m^{U}\right)} \leq A_{U} \exp \left(C_{U} T+A_{U} T^{-\nu_{U}}\right)\|\omega\|_{L^{1}\left(m^{U}\right)} \\
\omega \in C_{\mathrm{b}}\left(M ; \Lambda^{*}\left(T M^{*}\right)\right) \\
\left\|\mathbf{Q}_{T}^{U} \omega\right\|_{\mathrm{u}} \leq A_{U} \exp \left(C_{U} T+A_{U} T^{-\nu_{U}}\right)\|\omega\|_{L^{2}\left(m^{U}\right)}, \\
\omega \in C_{\mathrm{b}}\left(M ; \Lambda^{*}\left(T M^{*}\right)\right)
\end{array}
$$

In particular, for each $T \in(0, \infty), \mathbf{Q}_{T}^{U}$ admits a unique continuous extension as a bounded operator $\overline{\mathbf{Q}_{T}^{U}}$ on $L^{2}\left(m^{U} ; \Lambda^{*}\left(T M^{*}\right)\right)$, and $\overline{\mathbf{Q}_{T}^{U}}$ is both self-adjoint and Hilbert-Schmidt. In fact, $\overline{\mathbf{Q}_{T}^{U}}$ maps $L^{2}\left(m^{U} ; \Lambda^{*}\left(T M^{*}\right)\right)$ boundedly into $C_{\mathrm{b}}\left(M ; \Lambda^{*}\left(T M^{*}\right)\right)$, and

$$
\left\|\overline{\mathbf{Q}_{T}^{U}}\right\|_{\text {H.S. }}^{2} \leq 2^{d} m^{U}(M) A_{U} \exp \left(C_{U} T+A_{U} T^{-\nu_{U}}\right),
$$

where $\|\cdot\|_{H . S .}$ denotes the Hilbert-Schmidt norm.

Proof. Clearly (5.3) is a simple application of (2.12), and (4.19). Moreover, from (4.19) and the fact that $\mathbf{P}_{T}^{U}$ extends continuously to 
a contraction on $L^{2}\left(m^{U}\right)$, it is clear that $\mathbf{Q}_{T}^{U}$ admits a continuous extension as a bounded operator $\overline{\mathbf{Q}_{T}^{U}}$ on $L^{2}\left(m^{U} ; \Lambda^{*}\left(T M^{*}\right)\right)$. In addition, the self-adjointness of $\overline{\mathbf{Q}_{T}^{U}}$ is an easy consequence of the symmetry in (4.18); and, because (5.3) plus symmetry implies that each $\overline{\mathbf{Q}_{T}^{U}}$ maps $L^{2}\left(m^{U}\right)$ boundedly into $C_{\mathrm{b}}(M ; \mathbb{R})$, the set of $\omega \in L^{2}\left(m^{U} ; \Lambda^{*}\left(T M^{*}\right)\right)$ for which $\overline{\mathbf{Q}_{T}^{U}} \omega \in C_{b}\left(M ; \Lambda^{*}\left(T M^{*}\right)\right)$ is closed. Hence, since $\overline{\mathbf{Q}_{T}^{U}}$ maps $C_{b}\left(M ; \Lambda^{*}\left(T M^{*}\right)\right)$ into itself, it is clear that $\overline{\mathbf{Q}_{T}^{U}}$ takes the whole of $L^{2}\left(m^{U} ; \Lambda^{*}\left(T M^{*}\right)\right)$ boundedly into $C_{b}\left(M ; \Lambda^{*}\left(T M^{*}\right)\right)$.

Thus, all that remains is to check (5.4). For this purpose, choose an orthonormal basis $\left\{\omega_{n}: n \geq 1\right\}$ in $L^{2}\left(m^{U} ; \Lambda^{*}\left(T M^{*}\right)\right)$ so that $\omega_{n} \in$ $C_{\mathrm{c}}^{\infty}\left(M ; \Lambda^{*}\left(T M^{*}\right)\right)$ for each $n \geq 1$. Then each $\mathbf{Q}_{T}^{U} \omega_{n}$ is bounded and smooth, and, obviously,

$$
\sum_{n=1}^{N}\left\|\mathbf{Q}_{T}^{U} \omega_{n}\right\|_{L^{2}\left(m^{U}\right)}^{2} \leq m^{U}(M) \sup _{x \in M} \sum_{n=1}^{N}\left\|\left(\mathbf{Q}_{T}^{U} \omega_{n}\right)_{x}\right\|^{2} .
$$

Now let $x \in M$ be given, and choose $\left\{\eta_{k}: 1 \leq k \leq 2^{d}\right\} \subseteq \Omega_{\mathrm{c}}^{*}(M)$ so that $\left\{\left(\eta_{k}\right)_{y}: 1 \leq k \leq 2^{d}\right\}$ is an orthonormal basis in $\Lambda^{*}\left(T_{y} M^{*}\right)$ for each $y$ in an open neighborhood $W$ of $x$. Finally, choose $\left\{\psi_{r}: r \in(0,1]\right\} \subseteq$ $C_{\mathrm{c}}^{\infty}(W ;[0, \infty))$ so that $\psi_{r}$ is supported in the ball of radius $r$ around $x$ and $\int \psi_{r} d m^{U}=1$. Then

$$
\begin{aligned}
\sum_{n=1}^{N}\left\|\left(\mathbf{Q}_{T}^{U} \omega_{n}\right)_{x}\right\|^{2} & =\lim _{r \searrow 0} \sum_{k=1}^{2^{d}} \sum_{n=1}^{N}\left\langle\psi_{r} \eta_{k}, \mathbf{Q}_{T}^{U} \omega_{n}\right\rangle_{L^{2}\left(m^{U}\right)}^{2} \\
& =\lim _{r \searrow 0} \sum_{k=1}^{2^{d}} \sum_{n=1}^{N}\left\langle\mathbf{Q}_{T}^{U}\left(\psi_{r} \eta_{k}\right), \omega_{n}\right\rangle_{L^{2}\left(m^{U}\right)}^{2} \\
& \leq \lim _{r \searrow 0} \sum_{k=1}^{2^{d}}\left\|\mathbf{Q}_{T}^{U}\left(\psi_{r} \eta_{k}\right)\right\|_{L^{2}\left(m^{U}\right)}^{2} .
\end{aligned}
$$

Finally, by (5.3),

$$
\begin{aligned}
\left\|\mathbf{Q}_{T}^{U}\left(\psi_{r} \eta_{k}\right)\right\|_{L^{2}\left(m^{U}\right)}^{2} & \leq A_{U} \exp \left(C_{U} T+A_{U} T^{-\nu_{U}}\right)\left\|\psi_{r} \eta_{k}\right\|_{L^{1}\left(m^{U}\right)}^{2} \\
& \leq \exp \left(C_{U} T+A_{U} T^{-\nu_{U}}\right) .
\end{aligned}
$$

Hence, by letting $N \nearrow \infty$, we arrive at (5.4). q.e.d.

As we said, Lemma 5.2 is the heart of the matter. Nonetheless, there are still several annoying points which must be checked before we can prove Theorem 5.1. 
Lemma 5.5. Assume that $\omega, \omega^{\prime} \in \Omega^{*}(M) \cap L^{2}\left(m^{U} ; \Lambda^{*}\left(T M^{*}\right)\right)$.

(a) If $d \omega, \delta^{U} \omega^{\prime} \in L^{2}\left(m^{U} ; \Lambda^{*}\left(T M^{*}\right)\right)$, then

$$
\left\langle\omega^{\prime}, d \omega\right\rangle_{L^{2}\left(m^{U}\right)}=\left\langle\delta^{U} \omega^{\prime}, \omega\right\rangle_{L^{2}\left(m^{U}\right)} .
$$

(b) If $H^{U} \omega \in L^{2}\left(m^{U} ; \Lambda^{*}\left(T M^{*}\right)\right)$, then $d \omega, \delta^{U} \omega \in L^{2}\left(m^{U} ; \Lambda^{*}\left(T M^{*}\right)\right)$ and

$$
\left\langle\omega, H^{U} \omega\right\rangle_{L^{2}\left(m^{U}\right)}=\|d \omega\|_{L^{2}\left(m^{U}\right)}^{2}+\left\|\delta^{U} \omega\right\|_{L^{2}\left(m^{U}\right)}^{2} .
$$

Hence, if, in addition, $H^{U} \omega^{\prime} \in L^{2}\left(m^{U} ; \Lambda^{*}\left(T M^{*}\right)\right)$, then

$$
\begin{aligned}
\left\langle\omega^{\prime}, H^{U} \omega\right\rangle_{L^{2}\left(m^{U}\right)} & =\left\langle d \omega^{\prime}, d \omega\right\rangle_{L^{2}\left(m^{U}\right)}+\left\langle\delta^{U} \omega^{\prime}, \delta^{U} \omega\right\rangle_{L^{2}\left(m^{U}\right)} \\
& =\left\langle\omega, H^{U} \omega^{\prime}\right\rangle_{L^{2}\left(m^{U}\right)} .
\end{aligned}
$$

(c) If $H^{U} \omega \in L^{2}\left(m^{U} ; \Lambda^{*}\left(T M^{*}\right)\right)$, then

$$
d H^{U} \omega=0 \Longrightarrow H^{U} \omega=d \delta^{U} \omega \text {. }
$$

Proof. As in the proof of Lemma 2.15, construct a family $\left\{\psi_{R}: R>\right.$ $0\} \subseteq C_{\mathrm{C}}(M ;[0,1])$ so that $\psi_{R} \equiv 1$ on the ball $B(R)$ of radius $R$ around $o$ and $\left\|\operatorname{grad}_{x} \psi_{R}\right\|$ is bounded uniformly in $x \in M$ and $R>0$.

To prove (a), simply note that

$$
\left\langle\delta^{U} \omega^{\prime}, \psi_{R} \omega\right\rangle_{L^{2}\left(m^{U}\right)}=\left\langle\omega^{\prime}, d \psi_{R} \wedge \omega\right\rangle_{L^{2}\left(m^{U}\right)}+\left\langle\omega^{\prime}, \psi_{R} d \omega\right\rangle_{L^{2}\left(m^{U}\right)},
$$

and let $R \rightarrow \infty$.

Turning to (b), first note that

$$
\left\langle\omega, H^{U} \omega\right\rangle_{L^{2}\left(m^{U}\right)}=\lim _{R \rightarrow \infty}\left(\int_{M} \psi_{R}^{2}\left\langle\omega, d \delta^{U} \omega\right\rangle d m^{U}+\int_{M} \psi_{R}^{2}\left\langle\omega, \delta^{U} d \omega\right\rangle d m^{U}\right) .
$$

Second,

$$
\begin{aligned}
\int_{M} \psi_{R}^{2}\left\langle\omega, d \delta^{U} \omega\right\rangle d m^{U}= & \int_{M} \psi_{R}^{2}\left\|\delta^{U} \omega\right\|^{2} d m^{U} \\
& \left.+2 \int_{M} \psi_{R}\left\langle\operatorname{grad} \psi_{R}\right\lrcorner \omega, \delta^{U} \omega\right\rangle d m^{U} \\
\int_{M} \psi_{R}^{2}\left\langle\omega, \delta^{U} d \omega\right\rangle d m^{U}= & \int_{M} \psi_{R}^{2}\|d \omega\|^{2} d m^{U} \\
& +2 \int_{M} \psi_{R}\left\langle d \psi_{R} \wedge \omega, d \omega\right\rangle d m^{U}
\end{aligned}
$$


and, for each $\epsilon \in(0,1)$,

$$
\begin{aligned}
2\left|\int_{M} \psi_{R}\left\langle\operatorname{grad} \psi_{R}\right\lrcorner \omega, \delta^{U} \omega\right\rangle d m^{U} \mid \leq & \epsilon \int_{M} \psi_{R}^{2}\left\|\delta^{U} \omega\right\|^{2} d m^{U} \\
& \left.+\epsilon^{-1} \int_{M} \| \operatorname{grad} \psi_{R}\right\lrcorner \omega \|^{2} d m^{U}
\end{aligned}
$$

while

$$
\begin{aligned}
2\left|\int_{M} \psi_{R}\left\langle d \psi_{R} \wedge \omega, d \omega\right\rangle d m^{U}\right| \leq & \epsilon \int_{M} \psi_{R}^{2}\|d \omega\|^{2} d m^{U} \\
& +\epsilon^{-1} \int_{M}\left\|d \psi_{R} \wedge \omega\right\|^{2} d m^{U}
\end{aligned}
$$

Hence, after making a minor re-arrangement of terms and using the fact that

$$
\left.\int_{M}\left(\| \operatorname{grad} \psi_{R}\right\lrcorner \omega\left\|^{2}+\right\| d \psi_{R} \wedge \omega \|^{2}\right) d m^{U} \longrightarrow 0
$$

as $R \rightarrow \infty$, one sees that (5.7) follows. Moreover, given (5.7), (5.8) becomes a standard application of polarization.

Finally, to check $(\mathbf{c})$, observe that, because $\|d \omega\|$ and $\left\|H^{U} \omega\right\|$ are $m^{U}$-square integrable, $d H^{U} \omega=0$ implies that

$$
\int_{M} \psi_{R}^{2}\left\langle\delta^{U} d \omega, H^{U} \omega\right\rangle d m^{U}=2 \int_{M} \psi_{R}\left\langle d \omega, d \psi_{R} \wedge H^{U} \omega\right\rangle d m^{U} \longrightarrow 0
$$

as $R \rightarrow \infty$. At the same time, because $\delta^{U} \circ \delta^{U} \equiv 0$,

$$
\begin{aligned}
\int_{M} \psi_{R}^{2}\left\|\delta^{U} d \omega\right\|^{2} d m^{U}-\int_{M} \psi_{R}^{2}\left\langle\delta^{U} d \omega, H^{U} \omega\right\rangle d m^{U} \\
\quad=-\int_{M} \psi_{R}^{2}\left\langle\delta^{U} d \omega, d \delta^{U} \omega\right\rangle d m^{U} \\
\left.\quad=-2 \int_{M} \psi_{R}\left\langle\operatorname{grad} \psi_{R}\right\lrcorner \delta^{U} d \omega, \delta^{U} \omega\right\rangle d m^{U} \\
\quad \leq \frac{1}{2} \int_{M} \psi_{R}^{2}\left\|\delta^{U} d \omega\right\|^{2} d m^{U}+2 \int_{M}\left\|d \psi_{R} \wedge \delta^{U} \omega\right\|^{2} d m^{U} .
\end{aligned}
$$

Hence, because $\delta^{U} \omega \in L^{2}\left(m^{U} ; \Lambda^{*}\left(T M^{*}\right)\right)$, (5.9) follows from the preceding. q.e.d.

Lemma 5.10. $\left\{\overline{\mathbf{Q}_{t}^{U}}: t>0\right\}$ is a strongly continuous semigroup of self-adjoint contractions on $L^{2}\left(M ; \Lambda^{*}\left(T M^{*}\right)\right)$. Moreover, if $-\overline{H^{U}}$ denotes the generator of $\left\{\overline{\mathbf{Q}_{t}^{U}}: t>0\right\}$ and $\omega \in \Omega^{*}(M) \cap L^{2}\left(M ; \Lambda^{*}\left(T M^{*}\right)\right)$, 
then $\omega \in \operatorname{Dom}\left(\overline{H^{U}}\right)$ if and only if $H^{U} \omega \in L^{2}\left(m^{U} ; \Lambda^{*}\left(T M^{*}\right)\right)$, in which case $\overline{H^{U}} \omega=H^{U} \omega$. Finally, if $\omega \in L^{2}\left(M ; \Lambda^{*}\left(T M^{*}\right)\right)$, then, for each $t>$ $0, \overline{\mathbf{Q}_{t}^{U}} \omega$ is a bounded element of $\Omega^{*}(M) \cap \operatorname{Dom}\left(\overline{H^{U}}\right)$ and so $\overline{H^{U}} \overline{\mathbf{Q}_{t}^{U}} \omega=$ $H^{U} \overline{\mathbf{Q}_{t}^{U}} \omega$.

Proof. It is clear from (4.20) that, as $t \searrow 0, \overline{\mathbf{Q}}_{t}^{U} \omega \longrightarrow \omega$ in $L^{2}\left(m^{U} ; \Lambda^{*}\left(T M^{*}\right)\right)$, first for each $\omega \in \Omega_{c}^{*}(M)$ and then for all $\omega \in$ $L^{2}\left(m^{U} ; \Lambda^{*}\left(T M^{*}\right)\right)$. Hence, $\left\{\overline{\mathbf{Q}_{t}^{U}}: t>0\right\}$ is a strongly continuous semigroup of, as we already have noted, bounded self-adjoint operators.

Next note that if $\omega^{\prime} \in \Omega_{\mathrm{c}}(M)$, then Theorem 4.21 can be combined with (4.20) to see that

$$
H^{U} \mathbf{Q}_{t}^{U} \omega^{\prime}=-\partial_{t} \mathbf{Q}_{t}^{U} \omega^{\prime}=\mathbf{Q}_{t}^{U} H^{U} \omega^{\prime} \in L^{2}\left(m^{U} ; \Lambda^{*}\left(T M^{*}\right)\right) .
$$

Hence, if $\omega \in \Omega^{*}(M) \cap L^{2}\left(m^{U} ; \Lambda^{*}\left(T M^{*}\right)\right)$ and $H^{U} \omega \in L^{2}\left(m^{U} ; \Lambda^{*}\left(T M^{*}\right)\right)$, then, for all $\omega^{\prime} \in \Omega_{\mathrm{c}}^{*}(M)$,

$$
\begin{gathered}
\left\langle\omega^{\prime}, \omega\right\rangle_{L^{2}\left(m^{U}\right)}-\left\langle\omega^{\prime}, \overline{\mathbf{Q}_{t}^{U}} \omega\right\rangle_{L^{2}\left(m^{U}\right)}=\left\langle\omega^{\prime}, \omega\right\rangle_{L^{2}\left(m^{U}\right)}-\left\langle\mathbf{Q}_{t}^{U} \omega^{\prime}, \omega\right\rangle_{L^{2}\left(m^{U}\right)} \\
=\left\langle\int_{0}^{t} H^{U} \mathbf{Q}_{t}^{U} \omega^{\prime} d \tau, \omega\right\rangle_{L^{2}\left(m^{U}\right)}=\int_{0}^{t}\left\langle H^{U} \mathbf{Q}_{\tau}^{U} \omega^{\prime}, \omega\right\rangle_{L^{2}\left(m^{U}\right)} d \tau \\
=\int_{0}^{t}\left\langle\mathbf{Q}_{\tau}^{U} \omega^{\prime}, H^{U} \omega\right\rangle_{L^{2}\left(m^{U}\right)} d \tau=\int_{0}^{t}\left\langle\omega^{\prime}, \overline{\mathbf{Q}_{t}^{U}} H^{U} \omega\right\rangle_{L^{2}\left(m^{U}\right)} d \tau
\end{gathered}
$$

where, at the end, we first used (5.8) and then the self-adjointness of $\overline{\mathbf{Q}_{t}^{U}}$. But this means that we have now proved that

$$
\omega-\overline{\mathbf{Q}_{t}^{U}} \omega=\int_{0}^{t} \overline{\mathbf{Q}_{\tau}^{U}} H^{U} \omega d \tau
$$

which implies

$$
\lim _{t \searrow 0} \frac{\omega-\overline{\mathbf{Q}_{t}^{U}} \omega}{t}=H^{U} \omega \quad \text { in } L^{2}\left(m^{U} ; \Lambda^{*}\left(T M^{*}\right)\right),
$$

and this is equivalent to $\omega \in \operatorname{Dom}\left(\overline{H^{U}}\right)$ with $\overline{H^{U}} \omega=H^{U} \omega$. Conversely, if $\omega \in \Omega^{*}(M) \cap \operatorname{Dom}\left(\overline{H^{U}}\right)$, then, for all $\omega^{\prime} \in \Omega_{\mathrm{c}}^{*}(M)$,

$$
\begin{aligned}
\left\langle\omega^{\prime}, \overline{H^{U}} \omega\right\rangle_{L^{2}\left(m^{U}\right)} & =\left\langle\overline{H^{U}} \omega^{\prime}, \omega\right\rangle_{L^{2}\left(m^{U}\right)} \\
& =\left\langle H^{U} \omega^{\prime}, \omega\right\rangle_{L^{2}\left(m^{U}\right)}=\left\langle\omega^{\prime}, H^{U} \omega\right\rangle_{L^{2}\left(m^{U}\right)}
\end{aligned}
$$

since $\omega^{\prime} \in \operatorname{Hom}\left(\overline{H^{U}}\right)$ with $\overline{H^{U}} \omega^{\prime}=H^{U} \omega$. Hence, $\overline{H^{U}} \omega=H^{U} \omega^{\prime}$. 
Now let $\omega \in L^{2}\left(m^{U} ; \Lambda^{*}\left(T M^{*}\right)\right)$ be given. By the general theory (especially, Stone's Theorem) of semigroups of bounded, self-adjoint operators, we know that $t \in(0, \infty) \longmapsto \overline{\mathbf{Q}_{t}^{U}} \omega \in \operatorname{Dom}\left(\overline{H^{U}}\right)$ is differentiable and that $\partial_{t} \overline{\mathbf{Q}_{t}^{U}} \omega+\overline{H^{U}} \mathbf{Q}_{t}^{U} \omega=0$. Hence, in view of the preceding, what remains is to check that $\overline{\mathbf{Q}}_{t}^{U} \omega \in \Omega^{*}(M)$ for each $t>0$. But, by Lemma 5.2, we know that $\overline{\mathbf{Q}_{\frac{t}{2}}^{U}} \omega \in C_{b}\left(M ; \Lambda^{*}\left(T M^{*}\right)\right)$, and therefore $\overline{\mathbf{Q}_{t}^{U}} \omega=\mathbf{Q}_{\frac{t}{2}}^{U} \circ \overline{\mathbf{Q}_{\frac{t}{2}}^{U}} \omega \in \Omega^{*}(M)$ follows from Theorem 4.21.

Finally, we must still check that $\overline{\mathbf{Q}_{t}^{U}}$ is a contraction for each $t>0$. But, by (5.11) and (5.7), for any $\omega \in L^{2}\left(m^{U} ; \Lambda^{*}\left(T M^{*}\right)\right)$,

$$
\begin{aligned}
& \|\omega\|_{L^{2}\left(m^{U}\right)}^{2}-\left\|\overline{\mathbf{Q}_{T}^{U}} \omega\right\|_{L^{2}\left(m^{U}\right)}^{2} \\
& \quad=\int_{0}^{T}\left(\left\|d \overline{\mathbf{Q}_{t}^{U}} \omega\right\|_{L^{2}\left(m^{U}\right)}^{2}+\left\|\delta^{U} \overline{\mathbf{Q}_{t}^{U}} \omega\right\|_{L^{2}\left(m^{U}\right)}^{2}\right) d t \geq 0 .
\end{aligned}
$$

q.e.d.

Lemma 5.11. Assume that $\omega \in \Omega^{*}(M) \cap L^{2}\left(m^{U} ; \Lambda^{*}\left(T M^{*}\right)\right)$. Then, for each $t>0$,

$$
\begin{gathered}
d \omega \in L^{2}\left(m^{U} ; \Lambda^{*}\left(T M^{*}\right)\right) \Longrightarrow d \overline{\mathbf{Q}_{t}^{U}} \omega=\overline{\mathbf{Q}_{t}^{U}} d \omega \\
\delta^{U} \omega \in L^{2}\left(m^{U} ; \Lambda^{*}\left(T M^{*}\right)\right) \Longrightarrow \delta^{U} \overline{\mathbf{Q}_{t}^{U}} \omega=\overline{\mathbf{Q}_{t}^{U}} \delta^{U} \omega .
\end{gathered}
$$

Proof. We begin with the case in which $\omega \in \Omega^{*}(M) \cap \operatorname{Dom}\left(\overline{H^{U}}\right)$. Then, by (5.7),

$$
\begin{aligned}
& \left\|d \overline{\mathbf{Q}_{t}^{U}} \omega-d \omega\right\|_{L^{2}\left(m^{U}\right)}^{2}+\left\|\delta^{U} \overline{\mathbf{Q}_{t}^{U}} \omega-\delta^{U} \omega\right\|_{L^{2}\left(m^{U}\right)}^{2} \\
& =\left\langle\overline{\mathbf{Q}_{t}^{U}} \omega, H^{U} \overline{\mathbf{Q}_{t}^{U}} \omega\right\rangle_{L^{2}\left(m^{U}\right)}-2\left\langle\overline{\mathbf{Q}_{t}^{U}} \omega, H^{U} \omega\right\rangle_{L^{2}\left(m^{U}\right)}+\left\langle\omega, H^{U} \omega\right\rangle_{L^{2}\left(m^{U}\right)} \\
& =\left\langle\overline{\mathbf{Q}_{2 t}^{U}} \omega, H^{U} \omega\right\rangle_{L^{2}\left(m^{U}\right)}-2\left\langle\overline{\mathbf{Q}_{t}^{U}} \omega, H^{U} \omega\right\rangle_{L^{2}\left(m^{U}\right)}+\left\langle\omega, H^{U} \omega\right\rangle_{L^{2}\left(m^{U}\right)} \longrightarrow 0
\end{aligned}
$$

as $t \searrow 0$. In addition, because $d H^{U}=d \delta^{U} d=H^{U} d$,

$$
\partial_{t}\left(d \overline{\mathbf{Q}_{t}^{U}} \omega\right)=-d H^{U} \overline{\mathbf{Q}_{t}^{U}} \omega=H^{U}\left(d \overline{\mathbf{Q}_{t}^{U}} \omega\right) .
$$

Hence, if $\eta_{t}=d \overline{\mathbf{Q}_{t}^{U}} \omega-\overline{\mathbf{Q}_{t}^{U}} d \omega$, then $\left\|\eta_{t}\right\|_{L^{2}\left(m^{U}\right)} \longrightarrow 0$ and $\partial_{t} \eta_{t}=-H^{U} \eta_{t}$ for $t>0$. But, since

$$
\frac{d}{d t}\left\|\eta_{t}\right\|_{L^{2}\left(m^{U}\right)}^{2}=-2\left\langle\eta_{t}, H^{U} \eta_{t}\right\rangle_{L^{2}\left(m^{U}\right)} \leq 0
$$


this means that $\eta_{t}=0$ for all $t>0$. Exactly the same line of reasoning applies to $\delta^{U} \overline{\mathbf{Q}_{t}^{U}} \omega$

To handle the general case when $d \omega \in L^{2}\left(m^{U} ; \Lambda^{*}\left(T M^{*}\right)\right)$, we need to use the fact, coming from Lemma 5.2 and (5.7), that, for any $\eta \in$ $L^{2}\left(m^{U} ; \Lambda^{*}\left(T M^{*}\right)\right)$,

$\left\|d \overline{\mathbf{Q}_{t}^{U}} \eta\right\|_{L^{2}\left(m^{U}\right)}^{2}+\left\|\delta^{U} \overline{\mathbf{Q}_{t}^{U}} \eta\right\|_{L^{2}\left(m^{U}\right)}^{2}=\left\langle\overline{\mathbf{Q}_{t}^{U}} \eta, H^{U} \overline{\mathbf{Q}_{t}^{U}} \eta\right\rangle_{L^{2}\left(m^{U}\right)} \leq \frac{\|\eta\|_{L^{2}\left(m^{U}\right)}^{2}}{t}$

where the last inequality follows from elementary spectral theory. After combining this with the preceding, we see that, for each $t>0, \eta \rightsquigarrow$ $d \overline{\mathbf{Q}_{t}^{U}} \eta=\mathbf{Q}_{\frac{t}{2}}^{U} d \overline{\mathbf{Q}_{\frac{t}{2}}^{U}} \eta$ is a continuous map from $L^{2}\left(m^{U} ; \Lambda^{*}\left(T M^{*}\right)\right)$ into $C_{\mathrm{b}}\left(M ; \Lambda^{*}\left(T M^{*}\right)\right)$. Therefore, if we take $\left\{\psi_{R}: R>0\right\}$ as in the proof of Lemma 5.4, then

$$
d \overline{\mathbf{Q}_{t}^{U}} \omega=\lim _{R \rightarrow \infty} d \overline{\mathbf{Q}_{t}^{U}}\left(\psi_{R} \omega\right)=\lim _{R \rightarrow \infty} \overline{\mathbf{Q}_{t}^{U}} d\left(\psi_{R} \omega\right)=\overline{\mathbf{Q}_{t}^{U}} d \omega
$$

since $\psi_{R} \omega \longrightarrow \omega$ and

$$
d\left(\psi_{R} \omega\right)=d \psi_{R} \wedge \omega+\psi_{R} d \omega \longrightarrow d \omega
$$

in $L^{2}\left(m^{U} ; \Lambda^{*}\left(T M^{*}\right)\right)$. Again, the case when $\delta^{U} \omega \in L^{2}\left(m^{U} ; \Lambda^{*}\left(T M^{*}\right)\right)$ is handled by analogous reasoning. q.e.d.

Proof of Theorem 5.1. Set $\overline{A^{U}}=I-\overline{\mathbf{Q}_{1}^{U}}$. We begin by showing that

$$
\begin{aligned}
K_{U}^{*}(M) & =\operatorname{ker}\left(\overline{H^{U}}\right)=\operatorname{ker}\left(\overline{A^{U}}\right) \\
& =\left\{\omega \in \Omega^{*}(M) \cap L^{2}\left(m^{U} ; \Lambda^{*}\left(T M^{*}\right)\right): d \omega=0 \& \delta^{U} \omega=0\right\} \\
& \subseteq C_{\mathrm{b}}\left(M ; \Lambda^{*}\left(T M^{*}\right)\right) .
\end{aligned}
$$

To this end, first note that, by the second part of Lemma $5.10, K_{U}^{*}(M) \subseteq$ $\operatorname{ker}\left(\overline{H^{U}}\right)$. Second, observe that if $\omega \in \operatorname{ker}\left(\overline{H^{U}}\right)$, then $\partial_{t} \overline{\mathbf{Q}_{t}^{U}} \omega=-\overline{\mathbf{Q}_{t}^{U}}$ 。 $\overline{H^{U}} \omega=0$, and so $\omega=\overline{\mathbf{Q}_{1}^{U}} \omega$. Hence, $\operatorname{ker}\left(\overline{H^{U}}\right) \subseteq \operatorname{ker}\left(\overline{A^{U}}\right)$. Now suppose that $\omega \in \operatorname{ker}\left(\overline{A^{U}}\right)$. Then $\omega=\overline{\mathbf{Q}_{1}^{U}} \omega$, which, by the last part of Lemma 5.10 , means that $\omega \in \Omega^{*}(M)$. It also means that $\left.\omega=\overline{\left(\mathbf{Q}_{1}^{U}\right.}\right)^{n} \omega=$ $\overline{\mathbf{Q}_{n}^{U}} \omega$ for all $n \geq 1$. Since, by elementary spectral theory, $\overline{\mathbf{Q}_{n}^{U}} \omega$ tends to the orthogonal projection of $\omega$ onto $\operatorname{ker}\left(\overline{H^{U}}\right)$, we now know that $\omega \in \Omega^{*}(M) \cap \operatorname{ker}\left(\overline{H^{U}}\right)$. Hence, again by the second part of Lemma 5.10 , we have proved the first two equalities in (5.13). To prove the 
third, note that if $\omega \in \Omega^{*}(M) \cap L^{2}\left(m^{U} ; \Lambda^{*}\left(T M^{*}\right)\right)$ and $d \omega=0=\delta^{U} \omega$, then $\omega \in K_{U}^{*}(M)$. Conversely, if $\omega \in K_{U}^{*}(M)$, then, by (5.7), $d \omega=$ $0=\delta^{U} \omega$. Finally, by Lemma 5.2, $\omega \in K_{U}^{*}(M) \Longrightarrow \omega=\overline{\mathbf{Q}_{1}^{U}} \omega \epsilon$ $C_{\mathrm{b}}\left(M ; \Lambda^{*}\left(T M^{*}\right)\right)$.

We next let $\overline{E_{0}^{U}}$ denote orthogonal projection onto $K_{U}^{*}(M)$ and observe that, because $\overline{\mathbf{Q}_{1}^{U}}$ is compact and $\overline{H^{U}}$ is non-negative, the spectrum of $\overline{H^{U}}$ is discrete and therefore

$$
\left\langle\left(I-\overline{E_{0}^{U}}\right), \overline{H^{U}}\left(I-\overline{E_{0}^{U}}\right) \omega\right\rangle_{L^{2}\left(m^{U}\right)} \geq \alpha\left\|\left(I-\overline{E_{0}^{U}}\right) \omega\right\|_{L^{2}\left(m^{U}\right)}^{2}
$$

where $\alpha>0$ is the smallest strickly positive eigenvalue of $\overline{H^{U}}$. Hence, by elementary spectral theory, $\left\|\widehat{\mathbf{Q}_{t}^{U}} \circ\left(I-\overline{E_{0}^{U}}\right)\right\|_{\mathrm{op}} \leq e^{-\alpha t}$, and so

$$
\overline{G^{U}} \equiv \int_{0}^{\infty} \overline{\mathbf{Q}_{\tau}^{U}} \circ\left(I-\overline{E_{0}^{U}}\right) d \tau
$$

is well a defined operator with $\left\|\overline{G^{U}}\right\|_{o p} \leq \alpha^{-1}$. In fact, $\overline{G^{U}}$ maps $L^{2}\left(m^{U} ; \Lambda^{*}\left(T M^{*}\right)\right)$ onto $\operatorname{Dom}\left(\overline{H^{U}}\right)$ and

$$
\overline{H^{U}} \circ \overline{G^{U}}=I-\overline{E_{0}^{U}} .
$$

With these preparations, the rest of the proof goes as follows. Namely, by Lemma $5.2, \overline{A^{U}}$ is a self-adjoint, Fredholm operator. Hence, by (5.13), $K_{U}^{*}(M)$ is finite dimensional and the range of $\overline{A^{U}}$ coincides with $K_{U}^{*}(M)^{\perp}$. Now suppose that $\omega \in \Omega^{*}(M) \cap L^{2}\left(m^{U} ; \Lambda^{*}\left(T M^{*}\right)\right)$ with $d \omega=0$ is given, and set $\eta=\overline{E_{0}^{U}} \omega$. Then, $\omega-\eta=\overline{H^{U}} \zeta$, where $\zeta=\overline{G^{U}} \omega$. Because $\omega-\eta \in \Omega^{*}(M)$, elliptic regularity theory guanartees that $\zeta \equiv$ $\bar{G}^{U} \omega \in \Omega^{*}(M)$. At the same time, because $\omega-\eta \in L^{2}\left(m^{U} ; \Lambda^{*}\left(T M^{*}\right)\right)$, (5.7) and (5.9) guarantee that $\xi \equiv \delta^{U} \zeta \in L^{2}\left(m^{U} ; \Lambda^{*}\left(T M^{*}\right)\right)$ and that $d \xi=\omega-\eta$. Furthermore, because $\xi=\delta^{U} \zeta$, it is obvious that $\delta^{U} \xi=0$ and, by (5.6) and (5.13), that $\xi \perp K_{U}^{*}(M)$. Finally,

$\|\xi\|_{L^{2}\left(m^{U}\right)}^{2}=\left\|\delta^{U} \zeta\right\|_{L^{2}\left(m^{U}\right)}^{2}=\left\langle\overline{G^{U}}(\omega-\eta), \omega-\nu\right\rangle_{L^{2}\left(m^{U}\right)} \leq \alpha^{-1}\|\omega-\eta\|_{L^{2}\left(m^{U}\right)}^{2}$.

To prove the first of the uniqueness statements, observe that, by linearity, it suffices to know that 0 is the only $\eta \in K_{U}^{*}(M)$ which equals $d \xi$ for some $\xi \in \Omega^{*}(M) \cap L^{2}\left(m^{U} ; \Lambda^{*}\left(T M^{*}\right)\right)$. But this is obvious, since, by (5.6) and (5.13),

$$
\eta=d \xi \Longrightarrow\|\eta\|_{L^{2}\left(m^{U}\right)}^{2}=\langle\eta, d \xi\rangle_{L^{2}\left(m^{U}\right)}=\left\langle\delta^{U} \eta, \xi\right\rangle_{L^{2}\left(m^{U}\right)}=0
$$


To prove the second uniqueness statement, suppose that $\xi_{1}$ and $\xi_{2}$ were two elements of $\Omega^{*}(M) \cap L^{2}\left(m^{U} ; \Lambda^{*}\left(T M^{*}\right)\right)$ satisfying the stated properties, and set $\xi=\xi_{1}-\xi_{2}$. Then $\delta^{U} \xi=0, \xi \perp K_{U}^{*}(M)$, and $d \xi \in K_{U}^{*}(M)$. But, by the argument just given, $d \xi \in K_{U}^{*}(M) \Longrightarrow d \xi=0$. Hence, by (5.13), $\xi \in K_{U}^{*}(M) \cap K_{U}^{*}(M)^{\perp}$, and so $\xi=0$. q.e.d.

It may be useful to have the following alternative characterization of the $\xi$ appearing in the final statement of Theorem 5.1 when $\delta^{U} \omega \in$ $L^{2}\left(m^{U} ; \Lambda^{*}\left(T M^{*}\right)\right)$. Namely, in this case, $\xi$ is uniquely characterized by the facts that

$$
\xi \perp \operatorname{ker}\left(\overline{H^{U}}\right) \quad \text { and } \quad \xi-\overline{\mathbf{Q}_{1}^{U}} \xi=\int_{0}^{1} \overline{\mathbf{Q}_{\tau}^{U}} \delta^{U} \omega d \tau
$$

To see that there is at most one such $\xi$, observe that, in the notation of the preceding proof, (5.15) together with (5.13) say that $\xi$ is the unique element of $\operatorname{ker}\left(\overline{A^{U}}\right)$ such that $\overline{A^{U}} \xi=\int_{0}^{1} \overline{\mathbf{Q}_{\tau}^{U}} \delta^{U} \omega d \tau$. To see that the $\xi$ in the last statement of Theorem 5.1 satisfies (5.15), notice that, because $\delta^{U} \xi=0=\delta^{U} \eta, d \xi=\omega-\eta \Longrightarrow H^{U} \xi=\delta^{U} d \xi=\delta^{U} \omega$. Thus, $\partial_{t} \overline{\mathbf{Q}_{t}^{U}} \xi=-\overline{\mathbf{Q}_{t}^{U}} H^{U} \xi=-\overline{\mathbf{Q}}_{t}^{U} \delta^{U} \omega$, from which the second part of (5.15) is immediate.

\section{Application to De Rham cohomology}

Throughout this section we will be assuming that the Ricci curvature is bounded below, the Riemann curvature operator is bounded above (i.e., (4.4) holds), and that $U: M \longrightarrow[0, \infty)$ is a smooth function with the properties that

(i) $U$ has compact level sets.

(ii) There exist $C<\infty$ and $\theta \in(0,1)$ such that $\Delta U \leq C(1+U)$ and $\|\operatorname{grad} U\|^{2} \leq C e^{\theta U}$.

(iii) There exists an $\epsilon>0$ such that $\epsilon U^{1+\epsilon} \leq 1+\|\operatorname{grad} U\|^{2}$.

(iv) There exists a $B<\infty$ such that

$$
\left\langle X_{x}, \operatorname{hess}_{x} U X_{x}\right\rangle \geq-B\left\|X_{x}\right\|^{2}, \quad x \in M \text { and } X_{x} \in T_{x} M,
$$

where (cf. (3.21)), for $\mathfrak{f} \in \pi^{-1} x$ hess $_{x} U=\mathfrak{f} \circ \operatorname{Hess}_{\mathfrak{f}} \tilde{U} \circ \mathfrak{f}^{-1}$ is the Hessian of $U$. 
Clearly, these conditions are more than enough to guarantee that the conclusions drawn in Theorem 5.1 hold. In addition, they allow us to prove the following crucial result.

Lemma 6.2. For each $x \in M$, there is a unique path $t \in[0, \infty) \longmapsto$ $F_{t}^{U}(x) \in M$ such that $F_{0}(x)=x$ and $\frac{d}{d t} F_{t}^{U}(x)=-\operatorname{grad}_{F_{t}^{U}(x)} U$. In fact, $(t, x) \in[0, \infty) \times M \longmapsto F_{t}^{U}(x) \in M$ is a smooth map, $t \rightsquigarrow$ $U \circ F_{t}^{U}(x)$ is non-increasing, and, for each $t \in(0, \infty): F_{t}^{U}$ is diffeomorphic onto its image, its Jacobian determinant $J F$ is given by $\exp \left(-\int_{0}^{t} \Delta U\left(F_{\tau}^{U}\right) d \tau\right)$, and

$$
A_{U}(t) \equiv \sup _{x \in M} U\left(F_{t}^{U}(x)\right)<\infty .
$$

Finally, for each $(t, x) \in[0, \infty) \times M,\left(F_{t}^{U}\right)_{*}$, as a linear map from $T_{x} M$ to $T_{F_{t}^{U}(x)} M$, is bounded by (cf. (6.1)) $e^{B t}$.

Proof. The short time existence of $t \rightsquigarrow F_{t}^{U}(x)$ is guaranteed by general existence theory for solutions to O.D.E.'s, and the long time existence, as well as the asserted monotonicity, follows from the trivial observation that

$$
\frac{d}{d t} U\left(F_{t}^{U}(x)\right)=-\left\|\operatorname{grad}_{F_{t}^{U}(x)}\right\|^{2}
$$

In fact, by combining $\left(^{*}\right)$ with the condition $1+\|\operatorname{grad} U\|^{2} \geq \epsilon U^{1+\epsilon}$ in (iii) above, one quickly arrives at the asserted boundedness of $x \rightsquigarrow$ $U\left(F_{t}^{U}(x)\right)$ for each $t>0$. In addition, the expression for $J F_{t}^{U}$ is just an application of the usual relationship between the Jacobian determinant of a flow and the divergence of the vector field generating that flow, and the injectivity of $F_{t}^{U}$ is an easy consequence of uniqueness for O.D.E.'s. That is, if $F_{T}^{U}(x)=F_{T}^{U}(y)$ and $p(t)=F_{T-t}^{U}(x)$ while $q(t)=F_{T-t}^{U}(y)$ for $t \in[0, T]$, then $x=p(T)=q(T)=y$ because $\dot{p}(t)=\operatorname{grad}_{p(t)} U$ and $\dot{q}(t)=\operatorname{grad}_{q(t)} U$ for $t \in[0, T]$ and $p(0)=q(0)$. Finally, to obtain the bound on $\left(F_{t}^{U}\right)_{*}$, let $x \in M$ and $X_{x} \in T_{x} M$ be given, and set $X(t)=$ $\left(F_{t}^{U}\right)_{*} X_{x}$. Then, because the Lie derivative of $X(t)$ along $t \rightsquigarrow F_{t}^{U}(x)$ vanishes and therefore $\frac{D}{d t} X(t)=-\nabla_{X(t)} \operatorname{grad} U$,

$$
\begin{aligned}
\frac{d}{d t}\|X(t)\|^{2} & =2\left\langle\frac{D}{D t} X(t), X(t)\right\rangle=-2\left\langle\nabla_{X(t)} \operatorname{grad} U, X(t)\right\rangle \\
& =-2\left\langle X(t), \operatorname{hess}_{F_{t}(x)} U X(t)\right\rangle .
\end{aligned}
$$


Thus, by $(6.1), \frac{d}{d t}\|X(t)\|^{2} \leq 2 B\|X(t)\|^{2}$, from which the required estimate on $\left(F_{t}^{U}\right)_{*}$ follows immediately. q.e.d.

Because of Lemma 6.2 , we see that for any $\omega \in \Omega^{*}(M)$ the form $\left(F_{1}^{U}\right)^{*} \omega$ given by $\left(\left(F_{1}^{U}\right)^{*} \omega\right)_{x}=\left(F_{1}^{U}\right)^{*} \omega_{F_{1}^{U}(x)}$ is a bounded element of $\Omega^{*}(M)$. Moreover, because $d\left(F_{t}^{U}\right)^{*} \omega=\left(F_{t}^{U}\right)^{*} d \omega,\left(F_{t}^{U}\right)^{*}$ takes closed or exact forms into, respectively, closed or exact forms. In fact, because

$$
\begin{aligned}
\omega-\left(F_{1}^{U}\right)^{*} \omega= & \left.d\left(\int_{0}^{1}\left(F_{t}^{U}\right)^{*}(\operatorname{grad} U\lrcorner \omega\right) d t\right) \\
& \left.+\int_{0}^{1}\left(F_{t}^{U}\right)^{*}(\operatorname{grad} U\lrcorner(d \omega)\right) d t
\end{aligned}
$$

for all $\omega \in \Omega^{*}(M)$, it is clear that when $\omega \in \Omega^{*}(M)$ is closed, $\left(F_{1}^{U}\right)^{*} \omega$ is a bounded element of $[\omega]$ which is exact if $\omega$ is. Thus if we define $\Phi^{U} \omega$ to be the orthogonal projection of $\left(F_{1}^{U}\right)^{*} \omega$ onto $K_{U}^{*}(M)$, then, by Theorem 5.1, $\Phi^{U}$ is a well-defined, linear injection from $H^{*}(M)$ into $K_{U}^{*}(M)$. In particular, we have now proved most of the following.

Theorem 6.4. Under the conditions stated at the beginning of this section, $[\omega] \cap K_{U}^{*}(M)=\left\{\Phi^{U} \omega\right\}$ for each closed $\omega \in \Omega^{*}(M)$, and so the map $\Phi^{U}$, defined in the preceding paragraph, is linear isomorphism from $H^{*}(M)$ onto $K_{U}^{*}(M)$. Hence, for each $1 \leq q \leq d, \operatorname{dim}\left(H^{q}(M)\right)=$ $\operatorname{dim}\left(K_{U}^{q}(M)\right)<\infty$. In fact,

$$
\eta \in K_{U}^{*}(M) \Longrightarrow\|\eta+d \xi\|_{L^{2}\left(m^{U}\right)}^{2}=\|\eta\|_{L^{2}\left(m^{U}\right)}^{2}+\|d \xi\|_{L^{2}\left(m^{U}\right)}^{2}
$$

for all $\xi \in \Omega^{*}(M)$. Hence, for each closed $\omega \in \Omega^{*}(M), \Phi^{U} \omega$ can be characterized as the unique element of $[\omega] \cap L^{2}\left(m^{U} ; \Lambda^{*}\left(T M^{*}\right)\right)$ whose $L^{2}\left(m^{U}\right)$-norm is minimal.

Proof. In view of what we already know, all that remains is to prove (6.5), and clearly this comes down to showing that if $\xi \in \Omega^{*}(M)$ and $d \xi \in L^{2}\left(m^{U} ; \Lambda^{*}\left(T M^{*}\right)\right)$, then

$$
(\eta, d \xi)_{L^{2}\left(m^{U}\right)}=0
$$

To this end, first observe that, because $\eta$ is bounded and $\delta^{U} \eta=0$, the same argument which allowed us to prove (5.6) allows us to prove $\left(^{*}\right)$ when $\xi \in L^{1}\left(m^{U} ; \Lambda^{*}\left(T M^{*}\right)\right)$. Hence, we will be done if we can show that for any $\xi$ with $d \xi \in L^{2}\left(m^{U} ; \Lambda^{*}\left(T M^{*}\right)\right)$ there is a $\hat{\xi} \in \Omega^{*}(M) \cap$ $L^{1}\left(m^{U} ; \Lambda^{*}\left(T M^{*}\right)\right)$ such that $d \hat{\xi}=d \xi$. For this purpose, set

$$
\left.\hat{\xi}=\left(F_{1}^{U}\right)^{*} \xi+\int_{0}^{1}\left(F_{t}^{U}\right)^{*}(\operatorname{grad} U\lrcorner d \xi\right) d t .
$$


Then, because, by (6.3),

$$
\left.\xi-\hat{\xi}=d\left(\int_{0}^{1}\left(F_{t}^{U}\right)^{*}(\operatorname{grad} U\lrcorner \xi\right) d t\right)
$$

we know that $d \hat{\xi}=d \xi$. In addition, for each $T \in(0,1]$,

$$
\left.\left(F_{1}^{U}\right)^{*} \xi+\int_{T}^{1}\left(F_{t}^{U}\right)^{*}(\operatorname{grad} U\lrcorner d \xi\right) d t
$$

is bounded. Thus, it is enough for us check that, for some $T \in(0,1)$,

$$
\left.\sup _{t \in(0, T]} \|\left(F_{t}^{U}\right)^{*}(\operatorname{grad} U\lrcorner d \xi\right) \|_{L^{1}\left(m^{U}\right)}<\infty .
$$

To check $\left({ }^{* *}\right)$, first observe that, by the last part of Lemma 6.2 ,

$$
\left.\left.\|\left(F_{t}^{U}\right)^{*}(\operatorname{grad} U\lrcorner d \xi\right)\left\|\leq e^{d B t}\right\| \operatorname{grad}_{F_{t}} U\right\lrcorner(d \xi)_{F_{t} U} \| .
$$

At the same time, since $F_{t}^{U}$ is diffeomorphic onto its image, Jacobi's change of variables formula says that

$$
\begin{array}{rl}
\int_{M} \| \operatorname{grad}_{F_{t}} U & U(d \xi)_{F_{t}} \| d m^{U} \\
= & \int_{F_{t}^{U}(M)} \frac{\| \operatorname{grad} U\lrcorner d \xi \|}{\left(J F^{U}\right) \circ\left(F_{t}^{U}\right)^{-1}} e^{-U \circ\left(F_{t}^{U}\right)^{-1}} d \lambda_{M} \\
= & \left.\int_{F_{t}^{U}(M)} \| \operatorname{grad} U\right\lrcorner d \xi \| \exp \left(-U \circ\left(F_{t}^{U}\right)^{-1}\right. \\
& \left.+\int_{0}^{t} \Delta U\left(F_{s}^{U} \circ\left(F_{t}^{U}\right)^{-1}\right) d s\right) d \lambda_{M}
\end{array}
$$

where, in the last equality we have used the expression for $J F_{t}^{U}$ given in Lemma 6.2. To complete the proof from here, we use (ii) in our hypotheses about $U$ together with the fact that $s \rightsquigarrow U \circ F_{s}^{U}$ is nonincreasing, to see that

$$
\begin{array}{r}
\exp \left(-U \circ\left(F_{t}^{U}\right)^{-1}+\int_{0}^{t} \Delta U\left(F_{s}^{U} \circ\left(F_{t}^{U}\right)^{-1}\right) d s\right) \\
\leq e^{C t} \exp \left(-(1-C t) U \circ\left(F_{t}^{U}\right)^{-1}\right) .
\end{array}
$$


In particular, as long as $C t<1$, we now have the estimate

$$
\begin{aligned}
\left.\int_{M} \|\left(F_{t}^{U}\right)^{*}(\operatorname{grad} U\lrcorner d \xi\right) \| d m^{U} \\
\left.\quad \leq e^{(B+C) t} \int_{F_{t}{ }^{U}(M)} \| \operatorname{grad} U\right\lrcorner d \xi \| e^{-(1-C t) U} d \lambda_{M} \\
\quad \leq\left(\int_{M}\|\operatorname{grad} U\|^{2} e^{-(1-2 C t) U} d \lambda_{M}\right)^{\frac{1}{2}}\|d \xi\|_{L^{2}\left(m^{U}\right)} .
\end{aligned}
$$

In other words, all that we have to do is take $T \in(0,1]$ so that (cf. (i) in our hypotheses about $U) C T<\frac{1}{2}(1+\theta)$. q.e.d.

There are various applications which one can make of Theorem 6.4. For example, if $M_{1}$ and $M_{2}$ are two manifolds to which the last part of Theorem 6.4 applies and if $U_{1}$ and $U_{2}$ are allowable choice of the function $U$, then it is clear that $U\left(x_{1}, x_{2}\right)=U_{1}\left(x_{1}\right)+U_{2}\left(x_{2}\right)$ is an allowable choice for $M \equiv M_{1} \times M_{2}$ and that the associated $K_{U}^{*}(M)$ for $M$ will be the direct sum of the $K_{U_{1}}^{*}\left(M_{1}\right)$ and $K_{U_{2}}^{*}\left(M_{2}\right)$ for $M_{1}$ and $M_{2}$. Hence, since all these operators are non-negative, and therefore

$$
K_{U}^{q}(M) \simeq \sum_{q_{1}+q_{2}=q} K_{U_{1}}^{q_{1}}\left(M_{1}\right) \oplus K_{U_{2}}^{q_{2}}\left(M_{2}\right)
$$

the conclusion at the end of Theorem 6.4 leads to the Kunneth formula

$$
H^{q}(M) \simeq \sum_{q_{1}+q_{2}=q} H^{q_{1}}(M) \oplus H^{q_{2}}(M)
$$

for the De Rham cohomology groups.

Perhaps a more interesting application is the following Bochner-type vanishing theorem. In its statement, the functions $V^{\text {op }}$ and $V^{U}$ are defined on $M$ so that (cf. (3.19) and (3.20))

$$
\begin{aligned}
& V^{\mathrm{op}}(x)=\min \left\{\left\langle R^{\mathrm{op}} \omega_{x}, \omega_{x}\right\rangle: \omega_{x} \in \Lambda^{2}\left(T_{x} M\right) \text { with }\left\|\omega_{x}\right\|=1\right\} \\
& V^{U}(x)=\min \left\{\left\langle\operatorname{Ric}_{x} X_{x}+\nabla_{X_{x}} \operatorname{grad} U, X_{x}\right\rangle: X_{x} \in T_{x} M \text { with }\left\|X_{x}\right\|=1\right\} .
\end{aligned}
$$

Notice that both $V^{U}$ and $V^{\mathrm{op}}$ are locally Lipschitz continuous functions.

Theorem 6.5. If, under the hypotheses stated at the beginning of this section,

$$
\int_{M}\left(\|\operatorname{grad} \varphi\|^{2}+V^{U} \varphi^{2}\right) d m^{U}>0 \quad \text { for all } \varphi \in C^{1}(M ; \mathbb{R}) \backslash\{0\}
$$


then $H^{1}(M)=0$. Moreover, if

$$
\int_{M}\left(\|\operatorname{grad} \varphi\|^{2}+\left(V^{U}+V^{\mathrm{op}}\right) \varphi^{2}\right) d m^{U}>0, \quad \varphi \in C^{1}(M ; \mathbb{R}) \backslash\{0\}
$$

then $H^{q}(M)=0$ for each $2 \leq q \leq \operatorname{dim}(M)$. In particular, $H^{1}(M)=0$ if $V^{U}>0$ everywhere on $M$ and $\bigoplus_{q=2}^{\operatorname{dim}(M)} H^{q}(M)=0$ if $V^{U}+V^{\text {op }}>0$ everywhere on $M$.

Proof. Obviously, the last assertions are trivial applications of the first part of the theorem.

Turning to the proof of first part, define the family of operators $\left\{\mathbf{T}_{t}: t>0\right\}$ on $C_{\mathrm{b}}(M ; \mathbb{R})$ so that

$$
\mathbf{T}_{t} \varphi(x)=\mathbb{E}^{\mathbb{P}_{x}^{U}}\left[\exp \left(-\int_{0}^{t} V^{U}(p(\tau)) d \tau\right) \varphi(p(t))\right] .
$$

Again as a consequence of (4.4) and the reversibility of $\mathbb{P}_{x}^{U}$, it is easy to see that each $\mathbf{T}_{t}$ admits a unique extension $\overline{\mathbf{T}}_{t}$ as a bounded, self-adjoint operator on $L^{2}\left(m^{U}\right)$. Furthermore, by the Feynman-Kac formula, we know that the generator of $\left\{\overline{\mathbf{T}}_{t}: t>0\right\}$ acting on $\varphi \in C_{\mathrm{c}}^{2}(M ; \mathbb{R})$ is given by $L^{U} \varphi-V^{U} \varphi$. Finally, just as in the proof of Lemma 5.2, we can show that each $\overline{\mathbf{T}}_{t}$ is Hilbert-Schmidt and therefore compact. Thus, by elementary spectral theory, the strong minimum principle, and basic elliptic regularity theory, there either exists a $\varphi \in C^{1}(M ; \mathbb{R}) \cap L^{2}(M ; \mathbb{R})$ which violates $(6.8)$ or there exists a $\delta>0$ for which $\left\|\overline{\mathbf{T}}_{t}\right\|_{\text {op }} \leq e^{-\delta t}$. But in the latter case, for any $\omega \in K_{U}^{1}(M)$ we would have that

$$
\|\omega\|_{L^{2}\left(m^{U}\right)}=\left\|\overline{\mathbf{T}}_{t} \omega\right\|_{L^{2}\left(m^{U}\right)} \leq e^{-\delta t}\|\omega\|_{L^{2}\left(m^{U}\right)} \longrightarrow 0 \quad \text { as } t \rightarrow \infty .
$$

The conclusion drawn from (6.7) is proved by precisely the same line of reasoning. q.e.d.

Although they may be just as difficult to check in practice as the conditions given in Theorems 6.5, the following variation on those criteria there may be worth noting.

Corollary 6.8. Let everything be as in Theorem 6.5. Then a sufficient condition for $K_{U}^{1}(M)$ or $\bigoplus_{q=2}^{\operatorname{dim}(M)} K_{U}^{q}(M)$ to be trivial is that there exist a $\delta>0$ and a twice continuously differentiable, strictly positive function $u$ on $M$ such that, respectively,

$$
\Delta u-\langle\operatorname{grad} U, \operatorname{grad} u\rangle-V^{U} u \leq-\delta u
$$


or

$$
\Delta u-\langle\operatorname{grad} U, \operatorname{grad} u\rangle-\left(V^{\mathrm{op}}+V^{U}\right) u \leq-\delta u
$$

Proof. What we want to do is show that the existence of $u$ implies either (6.6) or (6.7). For this purpose, we will show that if $V: M \longrightarrow M$ is a continuous function which is bounded below and $u: M \longrightarrow(0, \infty)$ is a $C^{2}$ - function which satisfies $L^{U} u-V u \leq-\delta u$, then

$$
\delta\|\varphi\|_{L^{2}\left(m^{U}\right)}^{2} \leq \int_{M}\left(\|\operatorname{grad} \varphi\|^{2}+V^{U} \varphi^{2}\right) d m^{U}, \quad \varphi \in C_{\mathrm{C}}^{\infty}(M ; \mathbb{R})
$$

from which the desired conclusions are clear.

To prove (6.9), what we will do is check that

$$
\mathbb{E}^{\mathbb{P}_{x}^{U}}\left[\exp \left(-\int_{0}^{T} V(p(t)) d t\right) u(p(T))\right] \leq e^{-\delta T} u(x) .
$$

To see that (6.9) follows from (6.10), note that (6.10) implies that, for any $\varphi \in C_{\mathrm{C}}^{\infty}(M ;[0, \infty))$,

$$
\begin{array}{r}
\varlimsup_{T \rightarrow \infty} \frac{1}{T} \log \left(\int_{M} \varphi(x) \mathbb{E}^{\mathbb{P}}{ }_{x}^{U}\left[\exp \left(-\int_{0}^{T} V(p(t)) d t\right) \varphi(p(T))\right] m^{U}(d x)\right) \\
\leq-\delta
\end{array}
$$

which, by the Feynman-Kac formula and elementary spectral theory, implies (6.9).

Turning to the proof of $(6.10)$, take

$$
\zeta_{R}(p)=\inf \{t \geq 0: \rho(p(t)) \geq R\}
$$

for each $R>0$. Then, by a standard cut-off procedure, Doob's Stopping Time Theorem, and the product formula alluded to in the proof of (4.11),

$$
X_{R}(t, p) \equiv \exp \left(\int_{0}^{t \wedge \zeta_{R}(p)}(\delta-V(p(\tau))) d \tau\right) u\left(p\left(t \wedge \zeta_{R}\right)\right)
$$

is a non-negative, $\mathbb{P}_{x}^{U}$-supermartingale for every $x \in M$ and $R>0$. In particular, $\mathbb{E}^{\mathbb{P} x}\left[X_{R}(T, p)\right] \leq u(x)$, and so $(6.10)$ results after we let $R \rightarrow \infty$. q.e.d. 


\section{References}

[1] R. Bishop \& R. Crittenden, Geometry of manifolds, Academic Press, Pure Appl. Math. Ser. 15, 1964.

[2] J.-M. Bismut, The Witten complex and the degenerate Morse inequalities, J. Differential Geom. 23 (1986) 207-240.

[3] E. Bueler, The heat kernel with weighted Hodge Laplacian on noncompact manifolds, Trans. Amer. Math. Soc. 351 (1999) 683-713.

[4] E. Calabi, An extension of E. Hopf's maximum principle with an application to Riemannian geometry, Duke Math. J. 25 (1957) 45-56.

[5] T. Coulhon, Ultracontractive and Nash inequalities, J. Func. Anal. 141 (1996) $510-539$.

[6] E. B. Davies \& B. Simon, Ultracontractivity and the heat kernel for Schrödinger operators and the Dirichlet Laplacians, J. Fnal. Anal. 59 (1984) 335-395.

[7] K. D. Elworthy, X.-M. Li \& S. Rosenberg, Bounded and $L^{2}$-harmonic forms on universal covers, GAFA 8 (1998) 203-303, Birkhäuser, Basel.

[8] S. Kusuoka \& D. Stroock, Some boundedness properties of certain stationary diffusion semigroups, J. Func. Anal., 60 \# 2 (1985), 243-264

[9] P. Li \& S.-T.Yau, On the parabolic kernel of the Schrödinger operator, Acta. Math. 156 (1986) 153-201.

[10] M. Röckner \& F.-Y. Wang, Ultracontractivity and supercontractivity of Markov semigroups, Preprint.

[11] D. Stroock, On certain systems of parabolic equations, Comm. Pure Appl. Math. 23 (1970) 447-457.

[12] _ Probability theory, an analytic view, Cambridge Univ. Press, 1993, New York.

[13] An Introduction to the analysis of paths on a Riemmanian manifold, Amer. Math. Soc., Math. Surveys \& Monographs No. 74 Providence, RI, 2000.

[14] - The Ornstein-Uhlenbeck process on a Riemannian manifold, to appear in the Proceedings of the First ICCM.

[15] D. Stroock \& S. R. S. Varadhan, Multidimensional diffusion processes, Springer, Grundlehren, New York, Vol. 233, 1979.

[16] R. Schoen \& S.-T. Yau, Lectures on differential geometry, Internat. Press, Conf. Proc. \& Lecture Notes in Geom. \& Topology, Vol. I, 1994, Cambridge, MA.

[17] F.-Y. Wang, Functional inequalities for empty essential spectrum, J. Fnal. Anal. 170 (2000) 219-245. 
[18] E. Witten, Supersymmetry and Morse theory, J. Differential Geom. 17 (1982) 661-692.

[19] S.-T. Yau, On the heat kernel of a complete Riemannian manifold, J. Math. Pures Appl. Ser. 959 191-201.

Columbia University

Massachusetts Institute of Technology 